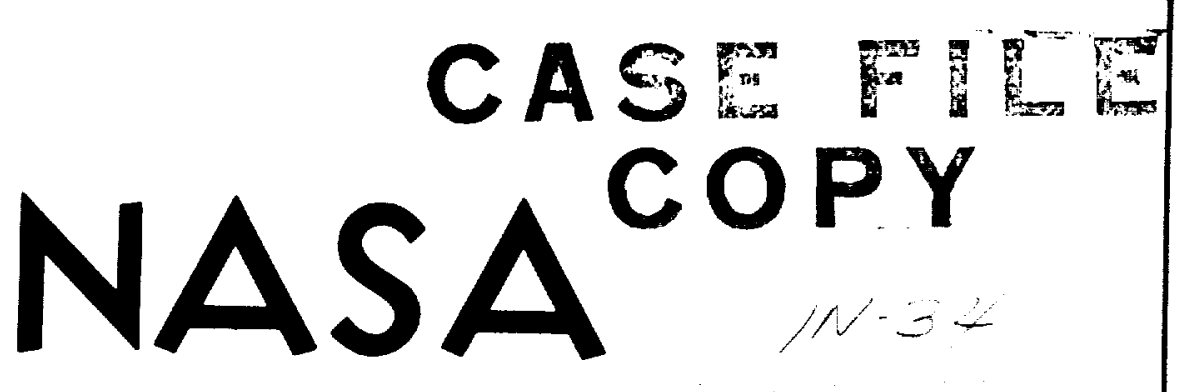

MEMORANDUM

USE OF A STANTON TUBE FOR SKIN-FRICTION MEASUREMENTS

By S. S. Abarbanel, R. J. Hakkinen, and L. Trilling

Massachusetts Institute of Technology

\title{
NATIONAL AERONAUTICS AND SPACE ADMINISTRATION WASHINGTON
}

March 1959 

MEMORANDUM 2-17-59W

USE OF A STANTON TUBE FOR SKIN-FRICTION MEASUREMENTS

By S. S. Abarbanel, R. J. Hakkinen, and L. Trilling

SUMMARY

A small total-pressure tube resting against a flat-plate surface was used as a Stanton tube and calibrated as a skin-friction meter at various subsonic and supersonic speeds. Laminar flow was maintained for the supersonic runs at a Mach number $M_{\infty}$ of 2 . At speeds between $M_{\infty}=1.33$ and $M_{\infty}=1.87$, the calibrations were carried out in a turbulent boundary layer. The subsonic flows were found to be in transition.

The skin-friction readings of a floating-element type of balance served as the reference values against which the Stanton tube was calibrated.

A theoretical model was developed which, for moderate values of the shear parameter $T$, accurately predicts the performance of the Stanton tube in subsonic and supersonic flows. A "shear correction factor" was found to explain the deviations from the basic model when $\tau$ became too large. Compressibility effects were important only in the case of turbulent supersonic flows, and they did not alter the form of the calibration curve.

The test Reynolds numbers, based on the distance from the leading edge and free-stream conditions, ranged from 70,000 to 875,000. The turbulent-boundary-layer Reynolds numbers, based on momentum thickness, varied between 650 and 2,300. Both laminar and turbulent velocity profiles were taken and the effect of pressure gradient on the calibration was investigated.

\section{INTRODUCTION}

In recent years there has been a renewed interest in the study of boundary-layer separation, especially at high speeds. Measurements of local shear forces are important in such investigations, but absolute skin-friction measuring devices of the floating-element type of balance are not suitable; the pressure gradients in the flow give rise to forces across the face of the floating element which may be of the same order of magnitude as the shear stresses to be measured. A device of much smaller characteristic length is needed to overcome this difficulty. 
Such an instrument is the Stanton tube (half-pitot surface tube). It consists essentially of an obstruction win a pressure orifice on its front face, the height of which is small compared with the boundarylayer thickness. In particular, a very smali total-pressure tube resting against the surface can serve as a Stanton tube. The ability of the Stanton tube to tolerate fairly steep pressure gradients is verified by recent studies of shock-wave-boundary-layer interaction ( ref. I) in which shear distributions were measured through the interaction region with the tube whose calibration is reported hereiz.

Until this investigation no reliable calibration of the Stanton tube had been made at supersonic speeds. Soine preliminary investigations were conducted in turbulent supersonic flow (ref. 2) and they yielded inconclusive results.

The Stanton tube was invented in 1920 and used by Stanton at the National Physical Laboratory in Teddington, Ingland. He built a halfpitot total-pressure tube for his studies of boundary layers in turbulent pipe flow (ref. 3). Fage and Falkner (ref. 4) used the Stanton tube in 1930 to measure skin friction on airfoil shapes. Taylor (ref. 5) summarized the existing results and performel additional experiments in 1938, essentially under Stokes flow conditions. He formulated the calibration problem in terms of two parameters and showed that all available data were in reasonable agreement. Further experiments, involving the use of the Stanton tube were rade by Fage and Sargent (ref. 6) and cope (ref. 7). In both investigations an attempt was made to measure supersonic skin friction using sibsonic calibration data. The validity of this extension of the calibration is doubtful.

A significant advance in the calibraticn of the Stanton tube is made possible by the availability of an absclute shear meter which replaces computed skin friction by measured values. Direct surface shear measurements were first taken in 1929 by Kempf (ref. 8) who used a floating element on the bottom of a barge to obtain skin-friction values at high Reynolds numbers. Not until 1951, however, were there any skin-friction meters suitable for high-speed work. Dhawan (ref. 9) in 1951 developed a floating-element balance: which could be used at high speeds. Hakkinen (ref. 2) used an improved version of Dwahan's instrument to obtain reference shear values and calibrated a Stanton tube in high-subsonic and low-supersonic flow. His results for subsonic speeds are in reasonable agreement with the older data. The supersonic measurements, however, show appreciable dev:ations and suggest compressibility effects. In 1954, on the basis of extensive experiments which suggested a unique pressure-shear relation, Preston (ref. 10) proposed the use of round pitot tubes resting agains; a flat surface as skinfriction meters. Stevenson (ref. 11) in $19^{\prime} .55$ obtained results which are in substantial agreement with Preston's dati. 
The object of the present investigation is to extend the Mach number range of the Stanton tube calibration, to determine when compressibility effects become important, and to propose a theoretical model to explain the calibration in various flow regimes.

This investigation was carried out at the Massachusetts Institute of Technology under the sponsorship and with the financial assistance of the National Advisory Committee for Aeronautics. The authors wish to express their thanks to the following persons: Dr. Jeffrey Tinkler who designed the basic floating element, Dr. Marten Landahl who graciously helped in making the element a working device, and Messrs. Kenneth Golden and Isaac Greber who performed the supersonic turbulent experiments.

\section{SYMBOLS}

$\begin{array}{ll}\text { A } & \text { proportionality constant, defined in equation (2l) } \\ \text { a } & \text { speed of sound } \\ \text { B } & \text { proportionality constant, defined in equation (22) } \\ c_{f} & \text { local skin-friction coefficient } \\ \mathrm{h} & \text { characteristic height of the Stanton tube } \\ \mathrm{M} & \quad \text { pressure } \\ \mathrm{P} & \mathrm{Stanton} \text { tube pressure } \\ \overline{\mathrm{P}}=\mathrm{p} / \mathrm{h}^{2} \quad & \\ \mathrm{P} & \end{array}$
$\mathrm{R}_{\mathrm{X}} \quad$ Reynolds number based on distance from leading edge and free- stream conditions
$\mathrm{R}_{\theta} \quad$ Reynolds number based on momentum-thickness and free-stream conditions


$\overline{\mathrm{T}}=\tau / \mathrm{h}^{2}$

$\mathrm{U}$

$U^{*}$ friction velocity in laminar subiayer, $\sqrt{\frac{\tau_{W}}{\rho_{W}}}$

$u=U /\left(\tau_{w} h / \mu_{w}\right)$

$u^{\prime}=U / \sqrt{\tau_{W} / \rho_{W}}$

$\mathrm{V}$

velocity in $\mathrm{Y}$-direction

$v=v /\left(T_{w} h / \mu_{w}\right)$

$v^{\prime}=V / \sqrt{T_{W} / \rho_{W}}$

$\mathrm{X}$

physical coordinate in streamwise: direction

$\mathrm{x}=\mathrm{x} / \mathrm{h}$

$\mathrm{Y}$ physical coordinate normal to $\mathrm{X}$

$\mathrm{y}=\mathrm{Y} / \mathrm{h}$

$\alpha$ ratio of laminar sublayer thickne:ss to probe height, $\epsilon / \mathrm{h}$

$\delta$ boundary-layer thickness

$\gamma \quad$ specific-heat ratio

$\epsilon \quad$ thickness of laminar sublayer

$\eta$

normal coordinate defined in equation (12a)

$\theta \quad$ momentum thickness of boundary liıyer, $\int_{0}^{\infty} \frac{\rho U}{\rho_{\infty} U_{\infty}}\left(I-\frac{U}{U_{\infty}}\right) d Y$

$\mu \quad$ coefficient of viscosity

$\rho$ density

$T \equiv \frac{T_{w} \rho_{w} h^{2}}{\mu_{w}^{2}}$ shear parameter 


$$
\begin{aligned}
& T_{W} \quad \text { shear stress at wall, } \mu_{W}\left(\frac{\partial U}{\partial Y}\right)_{Y=0} \\
& \tau_{\epsilon}=U^{*} \rho_{W} \epsilon / \mu_{W}
\end{aligned}
$$

Subscripts:

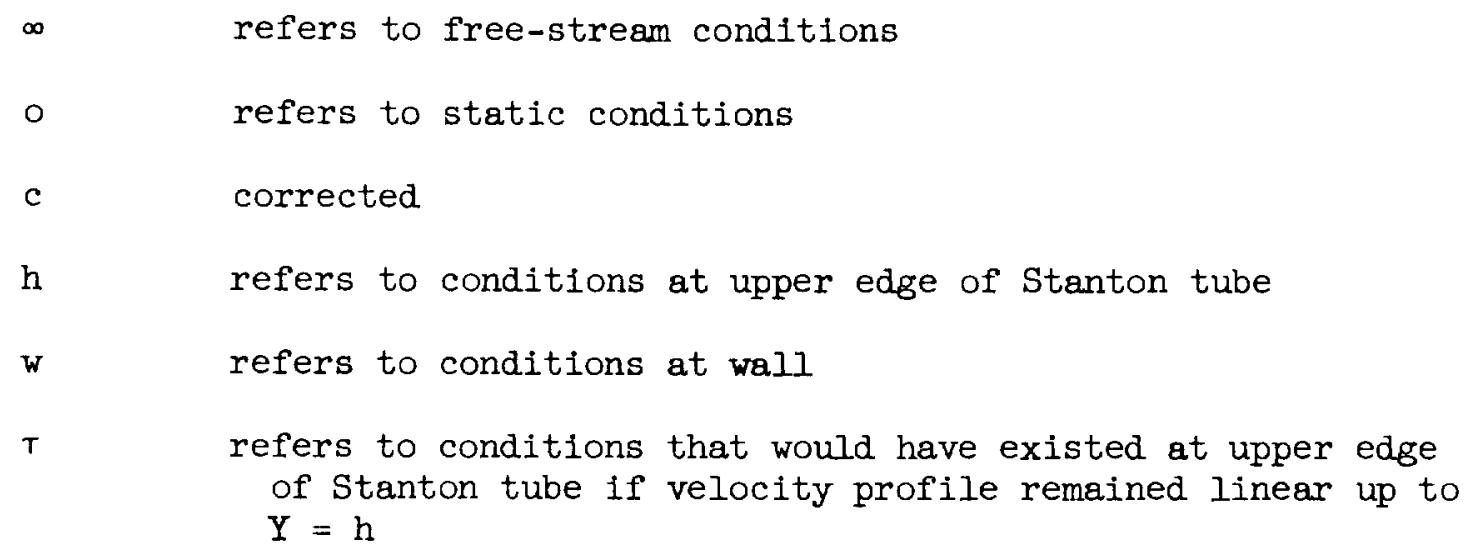

\section{EXPERIMENTAL APPARATUS}

\section{Wind Tunnels}

The experiments described herein were conducted in two wind tunnels. The subsonic and laminar supersonic runs were made in an 8- by 8-inch, continuous-flow supersonic tunnel in the M.I.T. Gas Turbine Laboratory. This is a vertical flow tunnel with a fixed nozzle of nominal Mach number 2 in which the air flows upwards.

The turbulent supersonic tests were made in a vertical wind tunnel having a $2-$ by $2 \frac{1}{2}$-inch closed circuit with variable Mach number and downward flow; this tunnel is also located in the Gas Thurbine Laboratory. The Mach number is varied by means of a sliding nozzle block (ref. 12). Wall boundary-layer removal ahead of the test plate is effected by a suction duct connected to a steam ejector.

The pressure level in both tunnels can be changed by means of steam ejectors. The stagnation pressure, which controls the test Reynolds number per unit length, can be varied from 0.2 to 2 atmospheres.

The two tunnels are shown in figures 1 and 2 . 


\section{Floating Element}

The floating-element skin-friction meter is shown in figure 3 . Two configurations, one for each wind tunne:-, were used in the present investigation. Both differed only slightly from the basic model used by Hakkinen (ref. 13). In a horizontal tunjel, movement of the floating element (fig. 3(a), part (A)) is due to the shear forces only in the absence of chordwise pressure gradient. In a vertical tunnel account must be taken of gravity. A counterweight (B) glued to the balance arm (C) serves to achieve a "neutral zero psint." Another departure from the basic model is the modification of the dashpot pamper (D). Originally the hollow cylinder (E) containel the damping fluid and a plunger which served both as a counterweight and as the dashpot piston. It was found, however, that surface tension and capillary suction due to the small clearance between the plunger and the cylinder walls prevented the use of that configuration.

In addition, the two modified configurations of the floating element differed from each other in two respects:

(1) The vertical air flow in the two tunnels was in opposite directions, so that whenever the device was transferred from one test section to the other it had to be "inverted."

(2) Since the flow in the variable Mach number tunnel was turbulent, stiffer springs (F) (fig. 3(a)) replazed the ones used in the larger tunnel in order to enable the instrunent to sustain bigger loads.

It should be noted that the whole device was designed to be as symmetrical as possible about two mutually perpendicular planes in order to minimize the effect of thermal deformations resulting from changes in flow recovery temperature.

Figures $4(a)$ and $4(b)$ give the calibration curves of the floating element under a static load. The readings are voltages induced in a Schaevitz coil (see fig. 5) by motion of the core due to the loading.

\section{Models}

The flat plate used in the 8 - by 8-inch tunnel (figs. 1 and 6), made of smoothly finished ground die steel, completely spanned the tunnel. Rubber gaskets were glued to the edges to prevent air leakage around the sides and to maintain two-dimensional flow. Forty-six static-pressure orifices gave chordwise anc. spanwise pressure distributions, which were recorded on a mercury marometer board. An ironconstantan-thermocouple junction located at, the same chordwise station as the element center line provided wall temperature readings. 
Figures 2 and 7 show the flat-plate model used in the $2-$ by $2 \frac{1}{2}-$ inch tunnel which was also made of ground die steel. The plate was firmly held flush against one of the tunnel walls with its sharp leading edge extending just beyond the boundary-layer-removal scoop. There were 13 static-pressure taps on the surface. A row of eight holes, spaced $1 / 4$ inch apart, located 0.30 inch from and parallel to the leading edge served as a boundary-layer trip. These holes were interconnected with a brass tube which had an outside lead equipped with a throttle. The pressure differential between the test section and the room induced a mass flow sufficient to trip the boundary layer. Two-dimensionality was maintained by O-rings inserted in channels machined along the sides of the plate. Wall temperature was measured with an iron-constanton thermocouple.

\section{Stanton Tube}

A sketch, a photograph, and a photomicrograph of the Stanton tube are shown in figures $8(a), 8(b)$, and $8(c)$, respectively. It was made of a hypodermic tube flattened and honed so that maximum probe-tip height and opening were 0.004 and 0.001 inch, respectively, as determined by measurements with a 62:1 optical comparator and a 30-power microscope. Both tunnels were equipped with traversing mechanisms which allowed motion streamwise and normal to the plate. The difference between the probe pressure and the appropriate static pressure could be recorded on either a mercury or a di-n-butyl phthalate U-tube manometer. The response time of the di-n-butyl manometer was of the order of 3 minutes.

The distance from the probe to the flat plate was determined visually by using a telescopic cathetometer (fig. 9). It is believed that the probe location could be determined well within \pm 0.001 inch. It was also found that even when the tube rested on the surface, as was required for the shear calibration, its lower edge did not quite touch the plate. The center line of the probe tip, when resting against the flat surface, was 0.0035 and 0.0028 inch away from the wall in the 8- by 8 -inch and 2- by $2 \frac{1}{2}$ - inch tunnels, respectively.

\section{EXPERTMENTAL RESULTS}

The experimental program consisted of obtaining direct shear measurements with the floating element and pressure readings with the 
Stanton tube. The skin-friction measurements served as the reference values against which the probe was calibrazed.

\section{Floating-Element Shear Measurements}

Figures 10 and 11 show the skin-friction-coefficient values measured in the 8 - by 8 -inch and the 2 - by $2 \frac{1}{2}$ - inch tunnels, respectively. The laminar reference curve used in figure 10 is calculated from Chapman and Rubesin's theory (ref. 14). The turbulent incompressible reference plcts are the semiempirical curves given by Von Kármán (ref. 15) and Coles (ref. 16). It is readily seen from figure 10 that the subsonic runs were in transition.

The laminar supersonic data (fig. 10) are represented rather well by a curve parallel to the theoretical one and 10 percent higher. The standard deviation due to scatter of the cata about the "best fit" value is 11 percent as computed by the least-square error method. The scatter is believed to be caused mainly by drift in tunnel conditions, which may affect the Reynolds numbers. Variations in the instrument null position also contributed to errors. The subject of gap effects is covered thoroughly by Hakkinen (ref. 2).

The turbulent skin-friction coefficients (fig. 1l) are plotted against $R_{\theta}$, the Reynolds numbers based oll the local momentum thickness. This is a more significant parameter than $R_{x}$ since the transition point is not well defined. Previous investigat .ons have not presented turbulent shear measurements in supersonic flor for values of $R_{\theta}$ below 1,500. As a matter of fact Coles (ref. 16) questions the validity of the turbulent-boundary-layer similarity conce ?t for $R_{\theta}=2,000$. The present data vary from $R_{\theta}=650$, which is very $n$ zar the critical Reynolds number, to $R_{\theta}=2,300$. The skin-friction measurements shown in figure 12 are in very good agreement with Hakkinen's data presented in reference 2 . The compressibility effects do not appear to be significant below $M_{\infty}=1.7$.

An additional source of experimental errors in turbulent shear measurements is the sensitivity of the monentum thickness

$\theta=\int_{0}^{\infty} \frac{\rho U}{\rho_{\infty} U_{\infty}}\left(1-\frac{U}{U_{\infty}}\right) d Y$ to the velocity-profile shape near the outer edge of the boundary layer. Typical turkulent velocity and momentum profiles are shown in figures 13 and 14 . 
Stanton Tube Calibration in Subsonic and

Laminar Supersonic Flow

Figure 12 is a logarithmic plot of $\overline{\mathrm{P}} \equiv \frac{\left(\mathrm{P}_{T}-\mathrm{P}_{\mathrm{O}}\right) \rho_{W}}{\mu_{W}^{2}}$ against
$\overline{\mathrm{T}} \equiv \frac{{ }_{W} \rho_{W}}{\mu_{W}^{2}}$ where $P_{\tau}, P_{O}, \quad T_{W}, \rho_{W}$, and $\mu_{W}$ are the probe pressure,

static pressure, wall shear stress, wall density, and viscosity, respectively. The data are represented rather well by the line

$$
\overline{\mathrm{P}}=0.006 \overline{\mathrm{T}} 5 / 6
$$

If $\overline{\mathrm{P}}$ and $\overline{\mathrm{T}}$ are nondimensionalized by division through by $h^{2}$, where $\mathrm{h}=0.01$ centimeter is the probe-tip height, the dimensionless calibration formula is found to be

$$
\mathrm{p}=2.79 \tau^{5 / 3}
$$

where $\mathrm{p} \equiv \overline{\mathrm{P}} / \mathrm{h}^{2}$ and $\tau \equiv \overline{\mathrm{T}} / \mathrm{h}^{2}$. The constant of proportionality is of order unity which is in agreement with the theoretical prediction (See section entitled "Theory of the Stanton Tube.")

As seen from figure 12 the calibration plot is an excellent representation of the subsonic points which exhibit a standard deviation of 3 percent from the curve. The supersonic points are also well described by the drawn line, with a scatter comparable to that shown in figure 10. This is to be expected since $T$ is essentially proportional to $c_{f}$. A plot of $p$ against $T$ computed from the Chapman-Rubes in formula shows a scatter of the order of 3 percent so that the main source of scatter in the supersonic measurements is probably in the measurements of skin friction.

\section{Stanton Tube Calibration in Turbulent Supersonic Flow}

The experimental results for the case of turbulent supersonic flow are not so clear cut or as amenable to analysis as the laminar and subsonic data. The best fit for the data in figure 15 is given by the line

$$
\bar{P}=0.390 \bar{T}^{4 / 3}
$$


or, reverting to the dimensionless form,

$$
p=7.95 \tau^{4 / 3}
$$

The scatter is considerably larger than that exhibited by the data in the plot of $c_{f}$ against $R_{\theta}$ (fig. ll). This would seem to indicate that not all the scatter in the stanton tube calibration plot is due to the random dispersion of experimental data. It is believed that because of the relatively narrow Mach number spectrun, $1.3<\mathrm{M}_{\infty}<1.9$, the deviations due to compressibility are of the same order as those induced by the random scatter and hence the difficulty n separating the two effects.

\section{Velocity Profile:;}

Two groups of velocity profiles were talien in the laminar supersonic runs and are shown in figure 16. Those: labeled group A were obtained at a distance of 3.0 inches from the leading edge at Reynolds numbers exceeding 400,000. Group B represent.s profiles measured 1.I inches from the leading edge at considerably lower Reynolds numbers. They are compared with the theoretical compressible boundary velocity profiles predicted by Chapman and Rubesin (ref. 14). The profiles of group A are in reasonable agreement with the predicted ones. Except for $\eta \leqq 1$, for which the experimental points coincide with the theoretical curve, the experimental data indicate an increase of about 10 to 12 percent in $\eta$. Bradfield (ref. 17), takirg profiles at a Mach number of 3 and a Reynolds number of 225,000, ncted the same discrepancy. In the present case, however, it is seen that the measured skin-friction stresses, given by the slope at $\eta=0$, are the same as the predicted ones.

The profiles of group B do not agree as well with the theoretical curve. These velocity profiles were taken at lower Reynolds numbers; uncertainties in probe location therefore gave rise to higher percentage errors. Another suurce of error was due to the fact that these velocity profiles were measured on the wedge part of the flat plate very near the leading edge ( 1.1 inches). Korobkir (ref. 18) has shown that recovery factors on the wedge are higher thar on an insulated plate. This effect is due to heat transfer from the lower wedge surface to the upper one, caused by the higher recovery temferature of the compressive flow at the lower surface. The same effect has also observed experimentally by Shoulberg, Hill, and Rivas (ref. 19). Had this effect been compensated for, the observed values of $\eta$ hould have been reduced somewhat. It should be noted, furthermore, that even group B profiles give the correct wall shear within 10 percent. 
Velocity profiles were taken for about half the turiulent runs. A typical profile is shown in figure 13. The slope at $Y=0$ was made to match the wall shearing stress as measured by the floating element. The momentum thickness of this profile is calculated from the plot of

$\frac{\rho U}{\rho_{\infty} U_{\infty}}\left(1-\frac{U}{U_{\infty}}\right)$ against $Y$ as shown in figure 13 . Notice that $\theta$ is about $1 / 8 \delta$, which is typical of turbulent boundary layers.

\section{Corrections Applied to Laminar Velocity Profiles}

In figure 16 it is observed that the points closest to the wall yield high values for $U / U_{\infty}$ when the data are reduced using the Bernoulli relation. It was decided to use the fact that the same total-head probe was used in measuring the profile and the skin friction. Near the wall the velocity proflle is linear and

$$
\frac{U}{U_{\infty}}=\frac{I}{U_{\infty}}\left(\frac{\tau_{W} Y}{\mu_{W}}\right)=\left(\frac{\mu_{W} Y}{\rho_{\infty} U_{\infty}}\right) \frac{\tau}{h^{2}}
$$

This becomes the corrected value $\left(U / U_{\infty}\right)_{c}$ when, for a given measured pressure, $T$ is obtained from the calibration curve.

This correction was applied to the velocity profiles of both groups A and B. The corrected values are indicated in figure 16 by flagged symbols. The success of the correction is most notable in the case of group A where the corrected points are seen to coincide with the theoretical profile. Because of the congestion of the many test points of group $B$ the shift due to the correction is not so clearly visible as it is in the case of group A, but all the corrected points of group B lie near the theoretical curve.

It is therefore suggested that the same correction is applicable even in the case where the probe had not previously been calibrated against an absolute skin-friction meter. The procedure would be modified somewhat. In the relation $\mathrm{p}=\mathrm{K} \tau^{5 / 3}, \mathrm{~K}$ is the constant of proportionality characteristic of the probe and is determined from a zeropressure-gradient velocity proflle by letting $T$ be the theoretical value. With this information the "high" points of other profiles can be corrected. This special procedure is most useful in cases where the profiles to be corrected are measured in regions for which a theoretical shear value is not easily available (e.g., decelerating flows over airfoils, etc.). 
THEORY OF THE STANION TUBE

The theory of the Stanton tube rests on the assumption that its characteristic height $h$ is small compared with the boundary-layer thickness $(\mathrm{h} / \delta \ll 1)$. Within this assumption there are two classes of problems. If $h / \delta$ is small enough, the pressure and velocity perturbations induced by the probe are confined to a very narrow band in which the boundary-layer velocity profile is lintsar and the mean flow in this sublayer is essentially incompressible. Tile other class consists of the cases where $h$ is still considerably smalier than $\delta$ but the perturbation field is no longer confined to the linear portion of the profile. These two classes of problems will be referred to as case I and case II,
respectively. As long as the mean flow in the sublayer is incompressible the
difference $P_{T}-\mathrm{P}_{\mathrm{O}}$ between the Stanton tube pressure and the wall static pressure depends only on the shearing stress $T_{W}$, the density $\rho_{W}$, the viscosity $\mu_{w}$, and the tube height $h$. As pointed out in references 2 and 10 dimensional analysis gives

$$
\Delta P \equiv P_{T}-P_{O}=T_{W} F\left(\frac{T_{w}{ }^{O} h^{c}}{\mu_{W}^{2}}\right)=T_{W} F(\tau)
$$

where, by the $\pi$-theorem, $\Delta P / \tau_{w}$ and $\tau \equiv \frac{{ }^{T} \rho_{w} h^{2}}{\mu_{w}^{2}}$ are the only two independent dimensionless parameters of the problem. In fact, for the case of the linear profile, $T$ is the Reynilds number based on the tube height $h$, and the velocity $U_{T}=\frac{T_{W} h}{\mu_{W}}$ at tlie edge of the tube. A dimensionless form of equation (4) is obtalned tlurough multiplication by $\left(\rho_{w} h^{2} / \mu_{w}^{2}\right)$ so that

$$
\frac{\left(P_{T}-P_{O}\right) \rho_{W} h^{2}}{\mu_{W}^{2}} \equiv p=\tau F\left(\tau^{*}=f(\tau)\right.
$$

The desired calibration curve is then given by $f(\tau)$. When the characteristic Reynolds number $T$ is small $(\tau \ll 1)$, the perturbation flow induced by the Stanton tube is a Stokes flow and $F(T)$ is a constant. Taylor (ref. 5) estimated that constant to bə 1.2 . 
When the free-stream Mach number increases sufficiently so that $M_{h}$, the Mach number at the upper edge of the tube, is appreciable, the fluid compressibility becomes important. In that case equation (5) must be rewritten as

$$
p=f\left(\tau, M_{h}\right)
$$

Let $M_{T}$ be the probe-tip Mach number in case $I$ (i.e., uniform shear flow). Then the parameter $M_{\tau}$ is related to the state of the fluid by the equation

$$
M_{\tau}^{2}=\frac{U_{\tau}^{2}}{a^{2}}=\frac{\left(\tau_{w} h / \mu_{W}\right)^{2}}{\gamma\left(P_{O} / \rho_{W}\right)}=\frac{\tau^{2}}{\gamma p_{O}}
$$

where $p_{O}$ is the dimensionless static pressure defined by $\frac{\mathrm{P}_{O} \rho_{w} h^{2}}{\mu_{w}^{2}}$.

Case I - The Stanton Tube in Uniform Shear Flow

An attempt is now made to derive an analytical expression for the calibration function $f(T)$ for the case in which the local Reynolds number $T$ is large. The theory assumes that the Stanton tube creates a boundary sublayer the thickness of which is of order $h\left(\frac{h}{6} \ll 1\right)$. In this inner sublayer the velocity profile is linear and the density and temperature are presumed to be constant. The present analysis for case I has been reported previously by Trilling and Hakkinen (ref. 20).

The dimensionless perturbation equations for the sublayer are obtained by perturbing the equation of a simple incompressible Couette flow in the manner of $\mathrm{Orr}$ and Sommerfeld. They are

$$
\begin{gathered}
y s_{x}+u_{x}+v_{y}=0 \\
y u_{x}+v+\frac{P_{x}}{T^{2}}=\frac{1}{T}\left[2 u+\frac{1}{3}\left(u_{x}+v_{y}\right)_{x}\right] \\
y v_{x}+\frac{P_{y}}{T^{2}}=\frac{1}{T}\left[\nabla^{2} v+\frac{1}{3}\left(u_{x}+v_{y}\right)_{y}\right]
\end{gathered}
$$


In these equations all lengths are made dimenisionless by division through by $h$, the probe-tip height; velocities, by d.vision through by $T_{w} h / \mu_{W}$, the mean velocity at the upper edge of the tulse; density $s$, by division through by $\rho_{\mathrm{w}}$. The pressure is as indicated in equation (5).

The perturbation flow is essentially isentropic. Using the isentropic relation $\mathrm{p} / \mathrm{s}^{\gamma}=$ Constant and the definition of $\mathrm{M}_{T}$, the equations become

$$
\begin{gathered}
\left.y M_{T}^{2} \frac{p_{x}}{T^{2}}+u_{x}+v_{y}=\right) \\
y u_{x}+v+\frac{p_{x}}{\tau^{2}}=\frac{1}{\tau}\left[\nabla^{2} u+\frac{1}{3}\left(u_{x}+v_{y}\right)\right] \\
y v_{x}+\frac{p_{y}}{T^{2}}=\frac{1}{T}\left[\nabla^{2} v+\frac{1}{3}\left(u_{x}+v_{y}\right)_{y}\right]
\end{gathered}
$$

The boundary conditions on the lower edge of the sublayer cannot be specified clearly since the details of the flow structure near the wall are very complicated. One condition to he selected is $\mathrm{v}(\mathrm{x}, 0)=0$. The component $u(x, 0)$ must vanish on the wal: up to the point $x=-L$ where the streamline $\psi=0$ leaves the wall; it vanishes on the surface of the tube; in the region $-\mathrm{L}<\mathrm{x}<0$ it is a function of $\mathrm{x}$ of order unity the precise form of which changes with ;ube geometry and is difficult to specify. All perturbations are boulded on the outer edge of the sublayer. Thus,

$$
\begin{gathered}
v(x, 0)=0 \\
u(x, 0) \equiv u_{0}(x)=0(I) \\
\lim _{y \rightarrow \infty} u, v<\infty
\end{gathered}
$$

Next PrandtI's boundary-layer argument $i$; applied to equations (8) to (10). It is desirable to stretch the normal coordinate and the normal velocity component so that in the resulting $x$ momentum equation all the variables and their derivatives are of order unity. The preceding 
argument is valid as long as $u_{0}(x)=0(1)$ and $T \gg 1$, so that equations ( 8 ) to (10) really represent a boundary-layer-type flow. Let the stretching be as follows:

$$
\left.\begin{array}{c}
\xi=x \\
\eta=y \tau^{k}
\end{array}\right\}
$$

where $k$ and $n$ are the "stretching" constants to be determined so that the transformed equations are compatible with the order-of-magnitude requirements.

The transformed equations are

$$
\begin{aligned}
& T^{-}(2+k+n) \eta_{T} M_{T}^{2} \bar{p}_{\xi}+\bar{u}_{\xi}+\bar{v}_{\eta}=0 \\
& \eta \tau^{1-3 k} \bar{u}_{\xi}+\tau^{1-3 k} \bar{v}+\tau^{-(1+2 k+n)} \bar{p}_{\xi}=\bar{u}_{\eta \eta}+\tau^{-2 k}\left(\frac{4}{3} \bar{u}_{\xi \xi}+\bar{v}_{\xi \eta}\right) \\
& \eta \tau^{-2 k} \bar{v}_{\xi}+\tau^{k-n-2} \overline{p \eta}=\tau^{-(k+1)} \bar{v}_{\xi \xi}+\tau^{1-k}\left(\frac{4}{3} \bar{v}_{\eta \eta}+\frac{1}{3} u_{\xi \eta}\right)
\end{aligned}
$$

If the inertia terms on the left-hand side of equation (14) are to balance the viscous terms, the exponents of $\tau$ must vanish in all the inertia terms in equation (14); that is,

$$
\begin{gathered}
1-3 k=0 \\
1+2 k+n=0
\end{gathered}
$$


from which

$$
\begin{gathered}
\mathrm{k}=\frac{1}{3} \\
\mathrm{n}=-\frac{5}{3}
\end{gathered}
$$

Substituting these values of $\mathrm{k}$ and $\mathrm{n}$ into the equations of motion, equations (13) to (15), gives

$$
\begin{aligned}
& \tau^{-2 / 3} \bar{M}_{\tau}{ }^{2} \eta \bar{p}_{\xi}+\bar{u}_{\xi}+\bar{v}_{T_{\xi}}=0 \\
& \eta \bar{u}_{\xi}+v+\bar{p}_{\xi}=\bar{u}_{\eta \eta}+\tau^{-2 / 3}\left(\frac{4}{\vdots} \bar{u}_{\xi \xi}+\bar{v}_{\xi \eta}\right) \\
& \tau^{-2 / 3} \eta \bar{v}_{\xi}+\bar{p}_{\eta}=\tau^{-2 / 3}\left(\frac{4}{3} \bar{v}_{\eta \eta}+\frac{1}{3} \bar{u}_{\xi \eta}\right)+\tau^{-4 / 3} \bar{v}_{\xi \xi}
\end{aligned}
$$

Since $T \gg 1$, all terms in $\tau^{-2 / 3}, T^{-4 / 3}$, and so forth are negligible in comparison with terms independent of $\tau$. It is seen from equation (18) that $\bar{p}_{\eta}=0\left(\tau^{-2 / 3}\right) \ll 1$, which is the usual boundary-layer result of a negligible normal pressure gradient across tye layer. The approximate sublayer equations are then

$$
\begin{gathered}
\bar{u}_{\xi}+\bar{v}_{\eta}=0 \\
\eta \bar{u}_{\xi}+\bar{v}+\bar{p}_{\xi}(\xi)=\bar{u}_{\eta \eta}
\end{gathered}
$$

Referring again to the transformation equati.n ( $12 b$ ) it is seen that

$$
\mathrm{p}=\overline{\mathrm{p}}^{-\mathrm{n}}=\overline{\mathrm{p}} \mathrm{T}^{5 / 3}
$$

and since $\bar{p}=O(1)$, the required form of $f(\tau)$ in equation (5) is found to be 


$$
p=A T 5 / 3
$$

where $A$ is a constant of proportionality of the order of 1 .

\section{Case II - The Nonlinear Profile}

As $T$ increases to very high values, either because of the large size of the Stanton tube or because of the high shear in a turbulent flow, it is possible that the probe, and hence the perturbation field, will extend beyond the linear portion of the velocity profile. In that case, the previous analysis which led to the 5/3-power law is invalid.

The physical situation is shown in the following sketch:

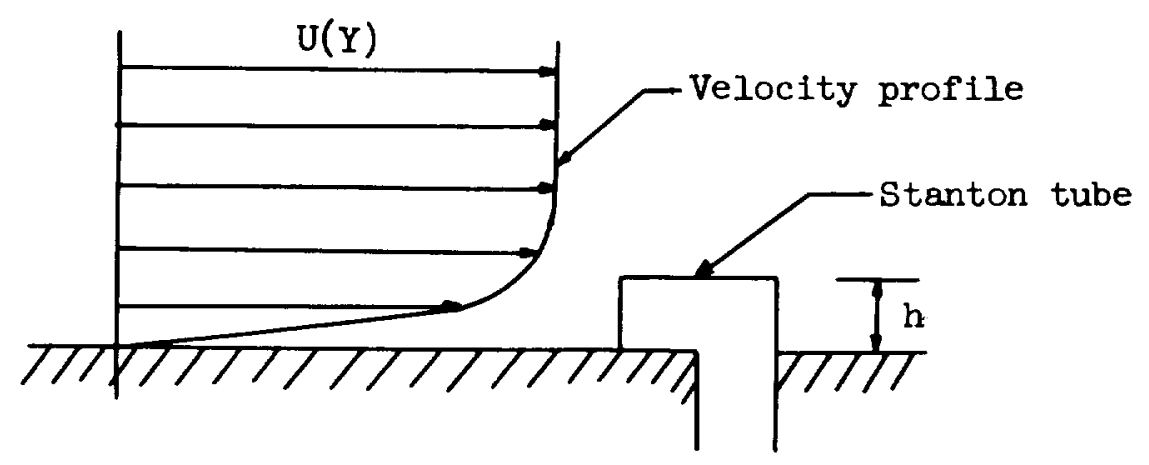

The calibration depends strongly on the shape of the profile. To simplify the analysis the actual physical situation is replaced by the following model:

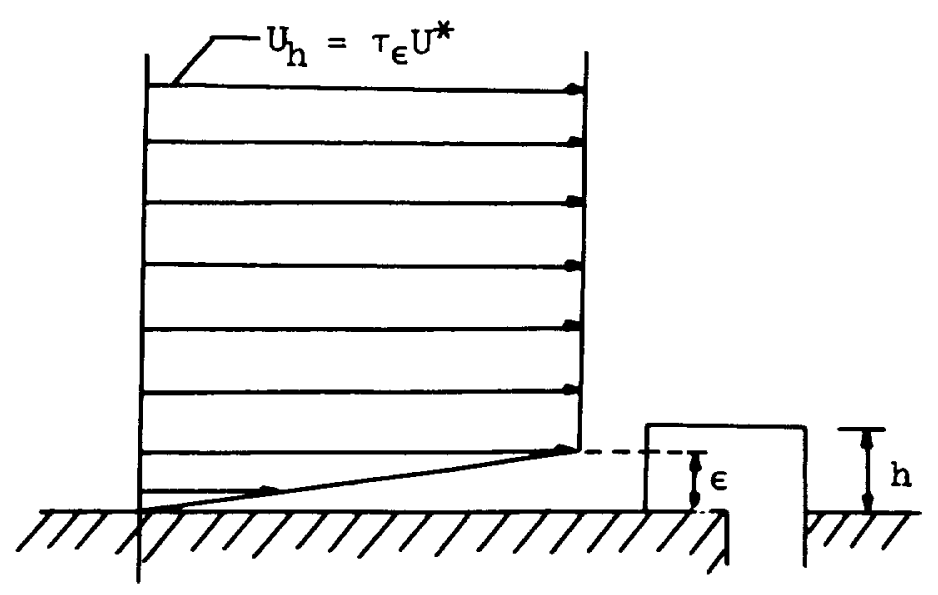


The constant velocity $U_{h}$ is taken to be proportional to the turbulent friction velocity $U^{*}=\sqrt{T_{W} / \rho_{w}}$. According to this model the form of the calibration function depends on the ratio of sublayer thickness to tube height, $\alpha=\epsilon / h$.

The problem can now be broken into its component parts, linear profile region and constant profile region. The effect of each can be found separately and then the two are superimposed with weighting functions $\alpha$ and $1-\alpha$ being multiplied inte the linear and constant profile contributions, respectively, as

$$
p=\alpha A f(\tau)+(1-\alpha) B g(\tau)
$$

where $A$ is the constant appearing in equation (2l), $g(\tau)$ is the characteristic function of the calibration curve for a constant velocity profile, and $\mathrm{B}$ is the constant of proportionality associated with $g(\tau)$. The justification of this procedure rests on the fact that the two terms in equation (22) add up to give the perturbation pressure at $\mathrm{Y}=\mathrm{h}$.

Next $g(\tau)$ is evaluated, $f(\tau)$ being found in the preceding section. The perturbation equations correspording to equations ( 8 ) to (10) are:

$$
\begin{gathered}
\tau \epsilon^{M^{*}} \frac{p_{x}}{\tau}+u_{x}^{\prime}+v_{y}^{\prime}=0 \\
\tau \epsilon^{u_{x}^{\prime}}+\frac{p_{x}}{\tau}=\frac{1}{\sqrt{\tau}}\left[\nabla^{2} u^{\prime}+\frac{1}{3}\left(u_{x}^{\prime}+v_{y}^{\prime}\right)_{x}\right] \\
\left.\tau_{\epsilon} v_{x}^{\prime}+\frac{p_{y}}{\tau}=\frac{1}{\sqrt{\tau}}\left[\nabla^{2} v^{\prime}+\frac{1}{3} u_{x}^{\prime}+v_{y}^{\prime}\right)_{y}\right]
\end{gathered}
$$

In these equations the nondimensionalizaticn is performed as before except that the velocity at the upper edge of the probe is taken to be $T_{\epsilon} U^{*}$ instead of $T_{w} h / \mu_{w} ; U^{*}=\sqrt{\tau_{w} / \rho_{w}}$ is the friction velocity connected with the sublayer of a turbulent boundary :ayer; and $\tau_{\epsilon}$ is a dimensionless universal constant for all turbulent boundary layers. It is the velocity (made dimensionless through division by $U^{*}$ ) of the flow at the outer edge of the laninar sublayer or the Reynolds number of the 
sublayer. The numerical value of $\tau_{\epsilon}$ is of the order of 20 . Also $M^{* 2}=U^{* 2} / a^{2}=\tau / r_{0}$.

If the same stretching procedure as before is followed, the transformed equations are

$$
\begin{gathered}
\tau_{\epsilon} \mathrm{M}^{*^{2}} \tau^{-(n+1)} \bar{p}_{\xi}+\bar{u}_{\xi}+\bar{v}_{\eta}=0 \\
\tau^{\frac{1}{2}-2 k} \tau_{\epsilon} \bar{u}_{\xi}+\tau^{-\left(\frac{1}{2}+n+2 k\right)} \bar{p}_{\xi}=\bar{u}_{\eta \eta}+\tau^{-2 k}\left(\frac{4}{3} \bar{u}_{\xi \xi}+\frac{1}{3} \bar{v}_{\xi \eta}\right) \\
\tau^{-k} \tau_{\epsilon} \bar{v}_{\xi}+\tau^{k-1} \bar{p}_{\eta}=\tau^{-1 / 2}\left(\tau^{-k} \bar{v}_{\xi \xi}+\frac{4}{3} \tau^{k} \bar{v}_{\eta \eta}+\frac{1}{3} \tau^{k} v_{\xi \eta}\right)
\end{gathered}
$$

from which $k=1 / 4$ and $\eta=-1$, so that $g(\tau)$ in equation (22) is given by

$$
g(\tau)=\tau
$$

and equation (22) becomes

$$
p=\alpha A T^{5 / 3}+(1-\alpha) B T
$$

Next it is observed that $\alpha$ can be expressed in the following manner:

$$
\alpha=\epsilon / h=\frac{\left(U^{*} \rho_{w} \epsilon / \mu_{w}\right)}{\left(U^{*} \rho_{w} h / \mu_{w}\right)}=\frac{\tau_{\epsilon}}{\sqrt{T}}
$$

Substituting equation (30) into equation (29) gives

$$
\mathrm{p}=\mathrm{A} \tau_{\epsilon} \frac{\tau^{5 / 3}}{\sqrt{\tau}}+\mathrm{BT}\left(1-\frac{\tau_{\epsilon}}{\sqrt{\tau}}\right)=\mathrm{A} \tau_{\epsilon} \tau^{7 / 6}+\mathrm{B}\left(\sqrt{\tau}-\tau_{\epsilon}\right) \sqrt{\tau}
$$


For large values of $\tau$ (say $\left.\tau>\tau_{\epsilon}^{2}\right)$ the first term on the righthand side of equation (31) predominates so that

$$
\mathrm{p} \approx \mathrm{Ar}_{\epsilon} \tau^{7 / 6}=\mathrm{A}^{\prime} \tau^{7 / 6}
$$

where $A^{\prime}$ is not of order unity but rather of order ${ }^{\top} \epsilon$.

This relation can be obtained in a different manner which is more useful for an extension of the theory to the compressible case. For $a \neq 0$, it can be deduced directly from equation (29) that for sufficiently high values of $T$

$$
\mathrm{p} \approx \alpha A \tau^{5 / 3}=\alpha p_{I}
$$

where $p_{I}$ is the calibration function for sase $I$. An evaluation of $\alpha$ may be made using an argument that reconciliss the actual velocity profile and the assumed model. Consider the geometry of the case shown in the following sketch:

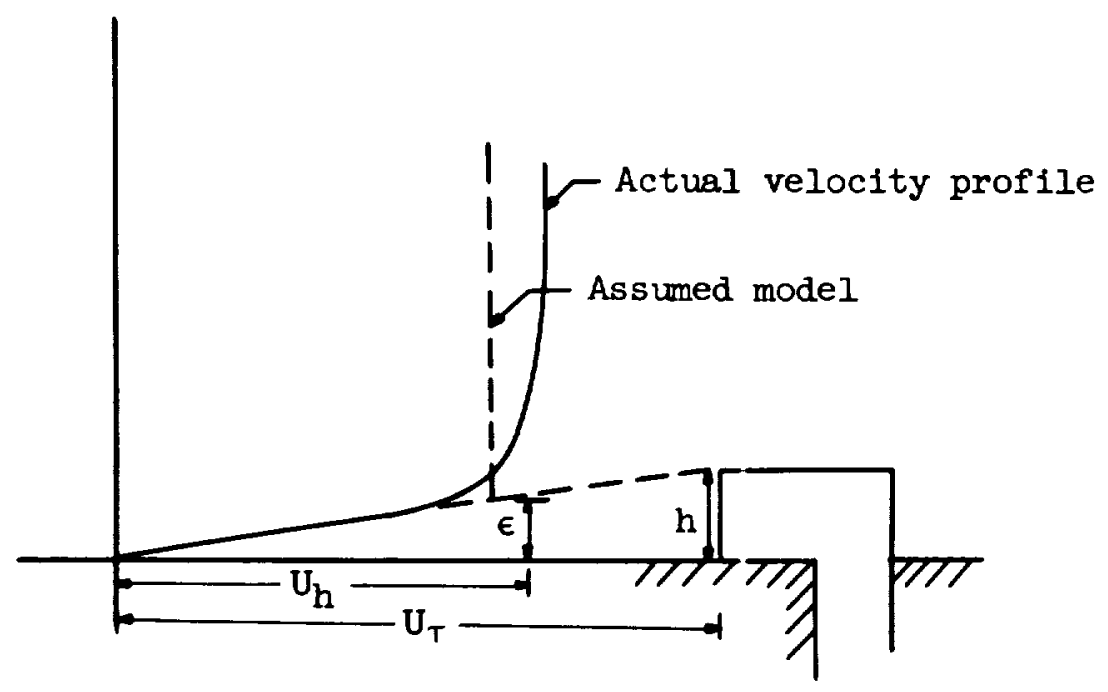

In this sketch $U_{h}$ is the actual velocity e.t the upper edge of the probe and $U_{T}$ is the velocity that would have exfsted at the upper edge of the probe if the profile had continued to be linear beyond $\epsilon$. Then,

$$
\alpha=\frac{\epsilon}{\mathrm{h}}=\frac{\mathrm{U}_{\mathrm{h}}}{\mathrm{U}_{\mathrm{T}}}
$$


so that equation (33) becomes

$$
p \equiv p_{I I}=\left(\frac{U_{h}}{U_{T}}\right) p_{I}=A \frac{U_{h}}{U_{\tau}} T^{5 / 3}
$$

where $p_{I I}$ is the desired calibration formula in case II.

It will now be demonstrated that $U_{h} / U_{T}$ is indeed proportional to $l / \sqrt{T}$. The actual velocity at $h$ is $U_{h}$. For large values of $T$, $\mathrm{U}_{\mathrm{h}}$ can be evaluated from the logarithmic law for turbulent velocity profiles. Van Driest (ref. 2l), for instance, gives for a smooth wall (using the present notation)

$$
\frac{U_{h}}{\sqrt{\tau_{W} / \rho_{W}}}=5.24+5.75 \log \sqrt{\tau}
$$

The velocity $U_{T}$ is defined as

$$
U_{T}=T_{w} h / H_{w}
$$

so that

$$
\frac{U_{h}}{U_{T}}=\frac{\sqrt{\tau_{W} / \rho_{W}}(5.24+5.75 \log \sqrt{\tau})}{\left(\tau_{W} h / \mu_{W}\right)}=\frac{5.24+5.75 \log \sqrt{T}}{\sqrt{T}}
$$

As long as $T$ is neither too small nor too large, say $10^{2}<\tau<10^{6}$, the behavior of $U_{h} / U_{T}$ is dominated by the denominator of equation (35); that is,

$$
\left(U_{h} / U_{\tau}\right) \propto \frac{1}{\sqrt{\tau}}
$$

Combining equations (34) and (35) gives

$$
\mathrm{p}_{I I}=\frac{\mathrm{U}_{\mathrm{h}}}{\mathrm{U}_{\mathrm{T}}} \mathrm{p}_{\mathrm{I}} \propto\left(\frac{1}{\sqrt{\tau}}\right) \mathrm{A} T^{5 / 3}=\mathrm{A}^{\prime \prime} \mathrm{T}^{7 / 6}
$$


It should be noted that $A^{\prime \prime}$ is not of order unity since the numerator of equation (35) is about 20 (1.e., of order ${ }^{\top} \epsilon$ ). Thus, equations (37) and (32) agree both in form and magnitude.

\section{COMPARISON OF PRESENT AND PREVIOUS EXFERTMENTS WITH THEORY}

Trilling and Hakkinen (ref. 20) gathered and presented systematically a wide variety of Stanton tube calibrations in subsonic flow. It is found that for $T<600$ the calibration functions follow the 5/3-power law. The present subsonic measurements fall within this range and agree with the previous results. The data of Preston (ref. 10) for $T>600$ and those of Fage and Sargent (ref. 6) for $T>200$ deviate appreciably from the 5/3-power law. In Preston's investization $p$ is found to be proportional to $\tau^{8 / 7}$. Fage and Sargent obtained a somewhat higher logarithmic slope for their measurements. It is suggested that in both instances the calibration falls within the case II classification and that the reported results are, within experimental errors, in agreement with the predicted 7/6-power law. In th? case of Preston's data the difference in the logarithmic slopes is less than 3 percent certainly within experimental accuracy. The constants of proportionality in the data of references 10 and 6 are 33 and 18, respectively. Allowing for differences in tube geometries, these values agree well with the predicted constant $A^{\prime}$ of order ${ }^{\top} \in$, which is about 20.

The present results in the laminar super zonic case seem to indicate that for moderate Mach number and laminar flow, compressibility effects are unimportant.

The turbulent supersonic results, yieldiing a 4/3-power law, can be explained by extending the results of the secion entitled "Case II The Nonlinear Profile." For the incompressib.e flow case the following shear correction was obtained:

$$
p \equiv p_{I I}=\left(\frac{U_{h}}{U_{T}}\right) p_{I}
$$

and since $U_{h} / U_{T}$ was found analytically to be proportional to $1 / \sqrt{T}$ the 7/6-power law resulted. If the argument :.s extended by analogy to the compressible case, the relation is expected to be of the form

$$
\mathrm{p}=\left(\frac{\mathrm{M}_{\mathrm{h}}}{\mathrm{M}_{\mathrm{T}}}\right) \mathrm{p}_{I}
$$


where $M_{h}$ is the actual Mach number at the upper edge of the probe, $\mathrm{M}_{T}$ is $\mathrm{T}^{2} / \mathrm{rp}_{\mathrm{o}}$, the Mach number which would have been obtained if the profile were linear up to $h$, and $p_{I}$ is the calibration formula for a linear profile.

Unfortunately, the Mach number profile for a supersonic turbulent boundary layer is not known analytically so that the dependence of $M_{h} / M_{T}$ on $T$ is not known. The alternative is to determine $M_{h} / M_{T}$ experimentally. Figure 17 presents a plot of $M_{T} / M_{h}$ computed from velocity profiles and static-pressure measurements. The data are well represented by the line

$$
M_{\tau} / M_{h}=0.18 \tau^{1 / 3}
$$

Substituting equation (40) into equation (39) gives

$$
p=\left(\frac{1}{0.18 \tau^{1 / 3}}\right)\left(2.79 \tau^{5 / 3}\right)=15.5 \tau^{4 / 3}
$$

as compared with the experimental calibration value of

$$
p=7.95 \tau^{4 / 3}
$$

Apparently two compressibility effects are encountered. Not only is the calibration power law changed through the Mach number dependence of the shear-correction factor $M_{h} / M_{\tau}$ but the proportionality constant is also affected directly. It is lower than expected from equation (32). Hakkinen (ref. 13) found at $\mathrm{M}_{\infty}=1.5$ that $\mathrm{p} / \mathrm{p}_{\text {subsonic }}=\mathrm{p} / \mathrm{p}_{\mathrm{I}}=2.3$. In the present investigation for the range of Mach numbers covered $\left(1.3<\mathrm{M}_{\infty}<1.9\right) \mathrm{p} / \mathrm{p}_{\mathrm{I}}=\frac{7.95}{2.79}=2.85$.

SUMMARY OF RESULTS

Available information on the calibration of the stanton tube as a skin-friction meter has been reviewed and analyzed. Additional experiments were performed in flow regions not covered in previous investigations. 
A theoretical model is developed to explain the subsonic results in the range $10<\tau<10^{3}$ of the dimensionless shear parameter. Th1s model is shown to be adequate also for laminar supersonlc flows. A shear correction applied to the basic model is used to predict the deviations occurring at higher values of $T$ when the perturbation field extends outside the linear portion of the boundary-layer velocity profile.

The main results are shown to be:

(1) In subsonic flow, for $10<\tau<10^{3}$, the dimensionless pressure is related to the shear parameter $T$ by an equation of the form

$$
\mathrm{p}=\mathrm{K} \mathrm{T}^{5 / 3}
$$

where $K$ is a constant of the order of unity which depends on the tube geometry only

(2) These results hold also for the cast of laminar supersonic flow for $M_{\infty} \leqq 2$ and $T \leqq 100$. The upper limits of $M_{\infty}$ and $T$ in the laminar supersonic case for which this rissult is still valid have not been established.

(3) In subsonic flow, for $\tau>600$ and for values of $h / \delta$ such that the probe sticks out of the linear portion of the profile, the calibration formula is

$$
\mathrm{p}=\mathrm{K}^{\prime} \tau^{7 / 6}
$$

where $K^{\prime}$ is of order $T_{\epsilon}$, the untversal turbulent Reynolds number based on the friction velocity $\sqrt{\tau_{w} / \rho_{w}}$ and isublayer thickness.

(4) In supersonic turbulent flow $\left(1.3<M_{\infty}<1.9\right)$ the calibration function is of the form

$$
p=K^{\prime \prime} \tau^{4 / 3}
$$

where $K^{\prime \prime}$ is smaller than $K^{\prime}$, apparently because of compressibility effects.

$\mathrm{All}$ the results concerning the form of ;he calibration function just summarized may be explained by the theo:etical model and the shear correction.

No compressibility effects were detected in the laminar supersonic tests. The range of Mach numbers in the turbulent supersonic experiments 
was insufficient to isolate the compressibility effects. Further investigations over a larger range of Mach numbers and shear parameter $T$ are needed before the compressibility effects can be fully understood.

A study to investigate the implication of the rapid density variations in the supersonic turbulent boundary layer is desired. It is possible that $T$, as defined herein using wall conditions only, is not the best-suited form of the shear parameter.

Massachusetts Institute of Technology, Cambridge, Mass., July 1, 1957. 


\section{REFERENCES}

1. Hakkinen, R. J., Greber, I., Trilling, L., and Abarbanel, S. S.: The Interaction of an Oblique Shock Wave With a Laminar Boundary Layer. NASA MEMO 2-18-59W, 1959.

2. Hakkinen, R. J.: Measurements of TurbuJent Skin Friction on a Flat Plate at Transonic Speeds. Ph.D. Thes is, C.I.T., 1954.

3. Stanton, T. E., Marshal, Dorothy, and Bryant, C. N.: On the Conditions at the Boundary of a Fluid in Turbulent Motion. Proc. Roy. Soc. (London), ser. A., vol. 97, no. A 687, Aug. 3, 1920, pp. 413-434.

4. Fage, A., and Falkner, V. M.: An Experimental Determination of Friction on the Surface of an Airfoil. R. \& M. No. 1315, British A.R.C., 1931.

5. Taylor, G. I.: Measurement With a Half-Pitot Tube. Proc. Roy. Soc. (London), ser. A., vol. 166, no. 927, June 16, 1938, pp. 476-481.

6. Fage, A., and Sargent, R. F.: Shock-Ware and Boundary-Layer Phenomena Near a Flat Surface. Proc. Roy. Soc. (London), ser. A. vol. 190, no. 1020, June 17, 1947, pp. 1-20.

7. Cope, W. F.: The Measurement of Skin Friction in a Turbulent Boundary Layer at a Mach Number of 2.5, Inc luding the Effect of a ShockWave. Proc. Roy. Soc. (London), ser. A, vol. 215, no. 1120, Nov. 6, 1952, pp. 84-99.

8. Kempf, G.: Neue Ergebnisse der Widerstendsforschung. Werft Reederei Hafen, Jahrg. 10, Heft 11, June 7, 19:9, pp. 234-239; Jahrg. 12, Heft 20, Oct. 15, 1931, pp. 247-253.

9. Dhawan, Satish: Direct Measurements of Skin Friction. NACA Rep. 1121, 1953. (Supersedes NACA TN 2567.)

10. Preston, J. H.: The Determination of Turbulent Skin Friction by Means of Pitot Tubes. Jour. R.A.S., vol. 5k, no. 518, Feb. 1954, pp. 109-121.

11. Stevenson, D. R.: On the Evaluation of Skin Friction of Turbulent Boundary Layers. M.E. Thesis, M.I.T. 1956.

12. Quigley, R. E., Lewis, S. D., and Mikami, K.: Description of a Variable Mach Number, Variable Reynolc.s Number Supersonic Test Section. TACP Rep. 16 (Contract No. fF 33(038)-22184), Dept. Aerc. Eng., M.I.T., Mar. 1955. 
13. Hrikinen, R. J.: Measurements of Turbulent Skin Friction on a Flat Plate at Transonic Speeds. NACA TN 3486, 1955.

14. Chapman, Dean R., and Rubesin, Morris W.: Temperature and Velocity Profiles in the Compressible Laminar Boundary Layer With Arbitrary Distribution of Surface Temperature. Jour. Aero. Sc1., vol. 16, no. 9, Sept. 1949, pp. 547-565.

15. Von Kármán, Th.: Turbulence and Skin Friction. Jour. Aero. Sci., vol. I, no. 1, Jan. 1934, pp. 1-20.

16. Coles Donald: The Problem of the Trubulent Boundary Layer. Z.f.a. Math. u. Phys., vol. V, fasc. 3, 1954, pp. 181-203.

17. Bradfield, W. S., DeCoursin, D. G., and Blumer, C. B.: The Effect of Leading-Edge Bluntness on a Laminar Supersonic Boundary Layer. Jour. Aero. Sci., vol. 21, no. 6, June 1954, pp. 373-382, 398.

18. Korobkin, Irving: Apparent Recovery Factors on the Leading Edge of a Flat Plate in Supersonic Laminar Flow. Jour. Aero. Sc1., vol. 20, no. 8, Aug. 1953, pp. 578-579.

19. Shoulberg, R. H., Hill, J. A. F., and Rivas, H. A., Jr.: An Experimental Determination of Flat Plate Recovery Factors for Mach Numbers Between 1.30 and 3.14. Jour. Aero. Sci., vol. 21, no. 11, Nov. 1954, pp. 763-771.

20. Trilling, L., and Hakkinen, R. J.: The Calibration of the Stanton Tube as a Skin-Friction Meter. 50 Jahre Grenzschichtforschung, H. Görtler and W. Tollmien, eds., Friedr. Vieweg \& Sohn (Braunschweig), 1955, pp. 201-209.

21. Van Driest, E. R.: On Turbulent Flow Near a Wall. Jour. Aero. Sc1., vol. 23, no. 11, Nov. 1956, pp. 1007-1011. 


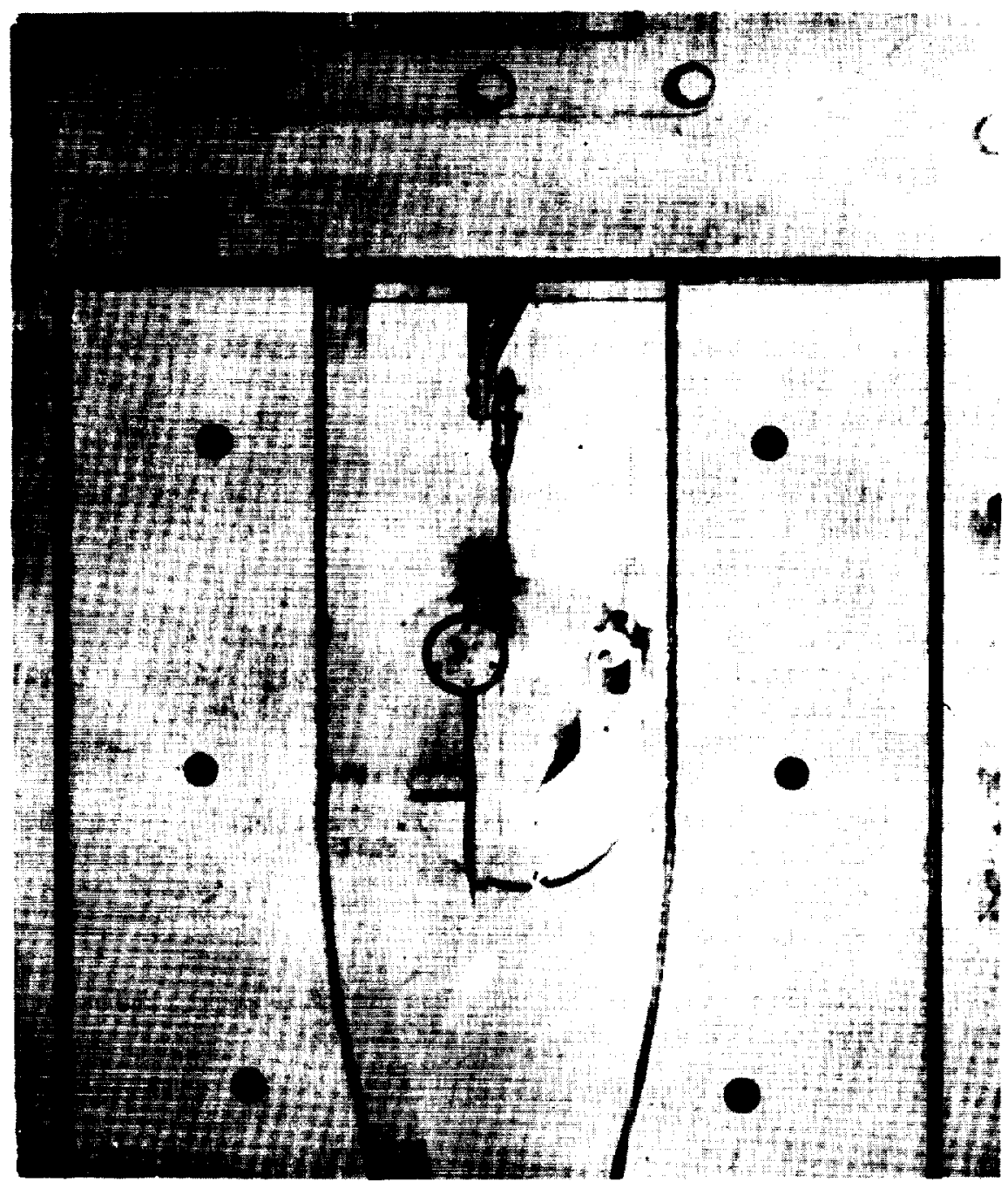

L-58-3992

Figure 1.- Flat plate mounted in 8 - by 8 -inch supersonic wind tunnel. 


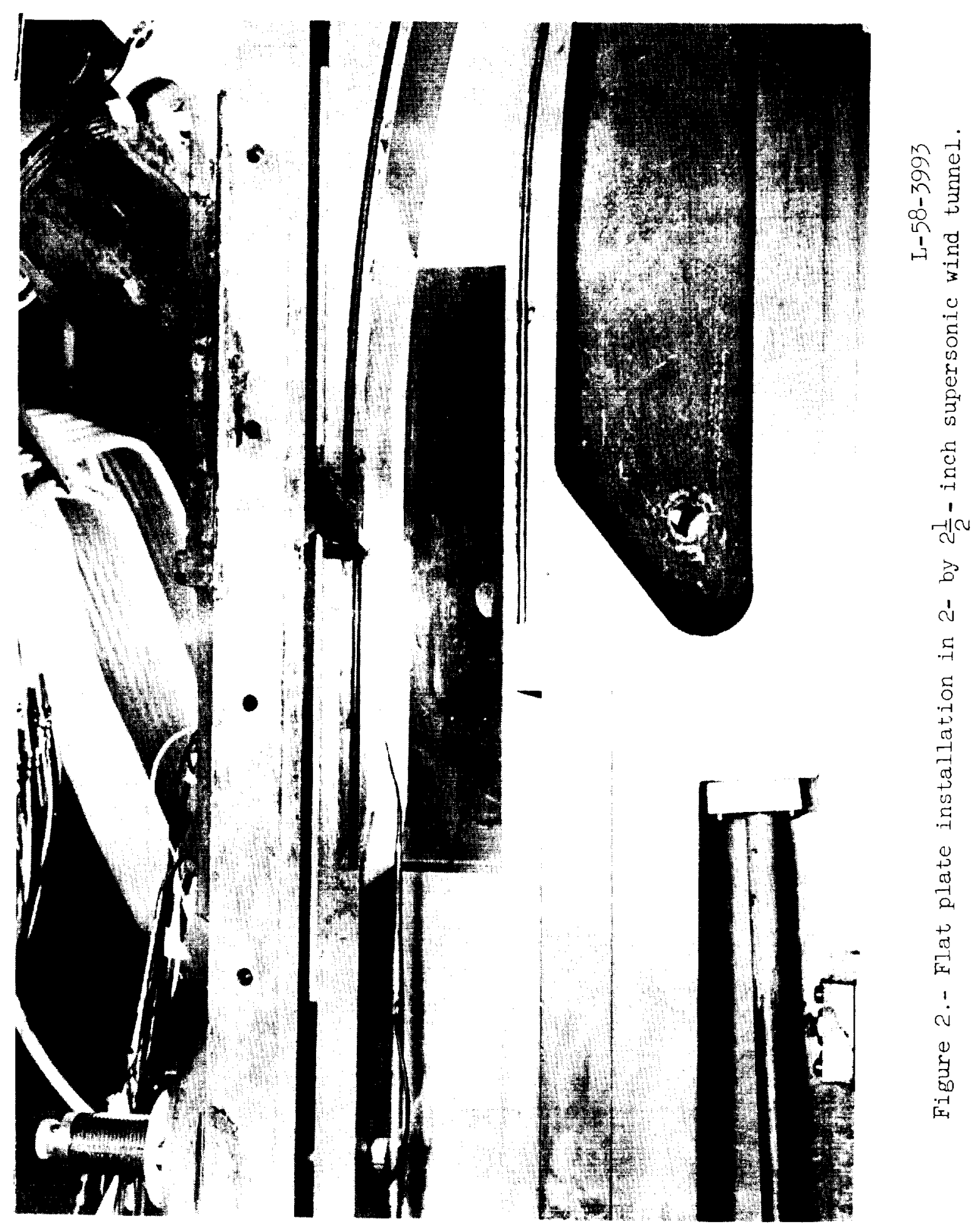




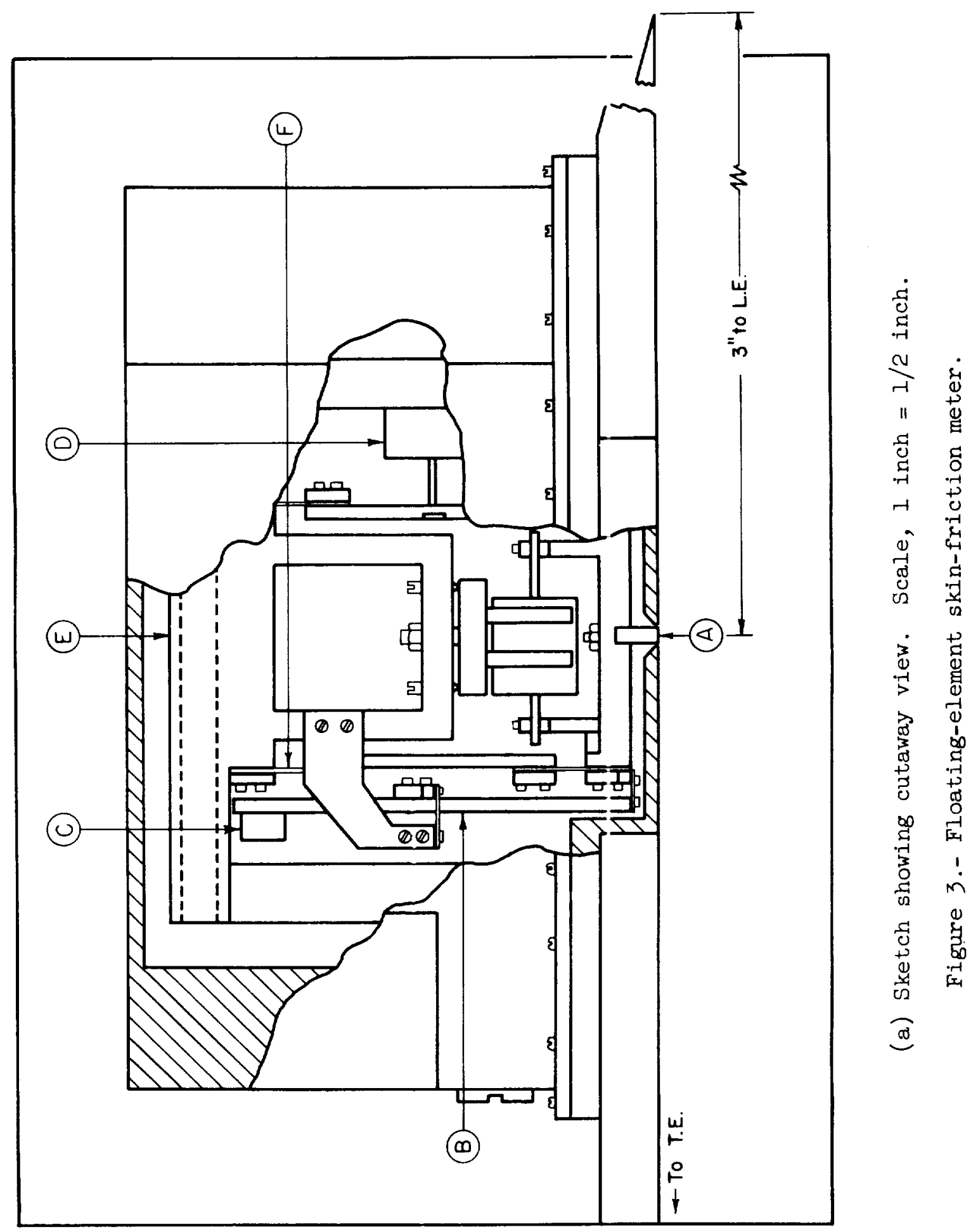




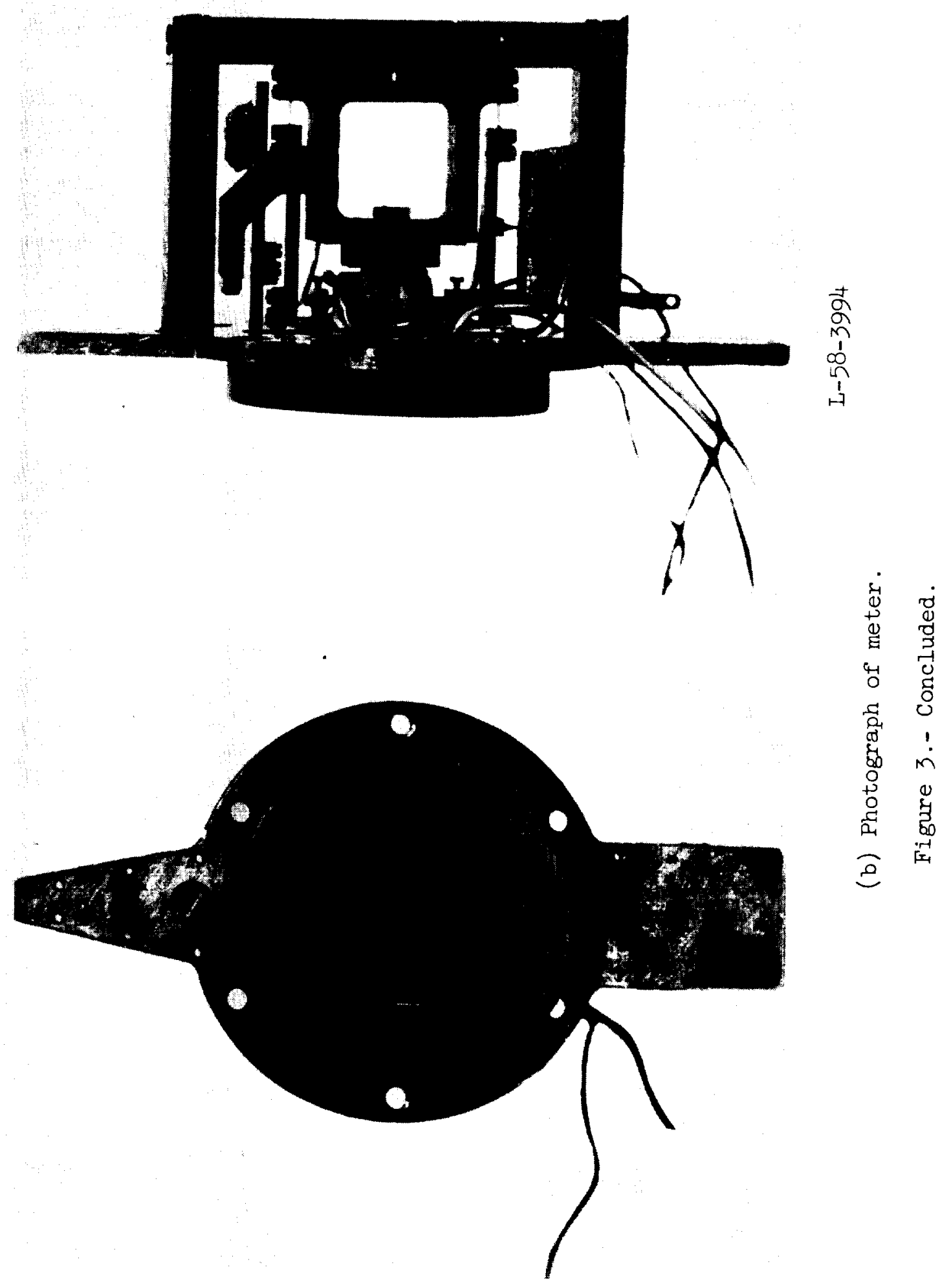




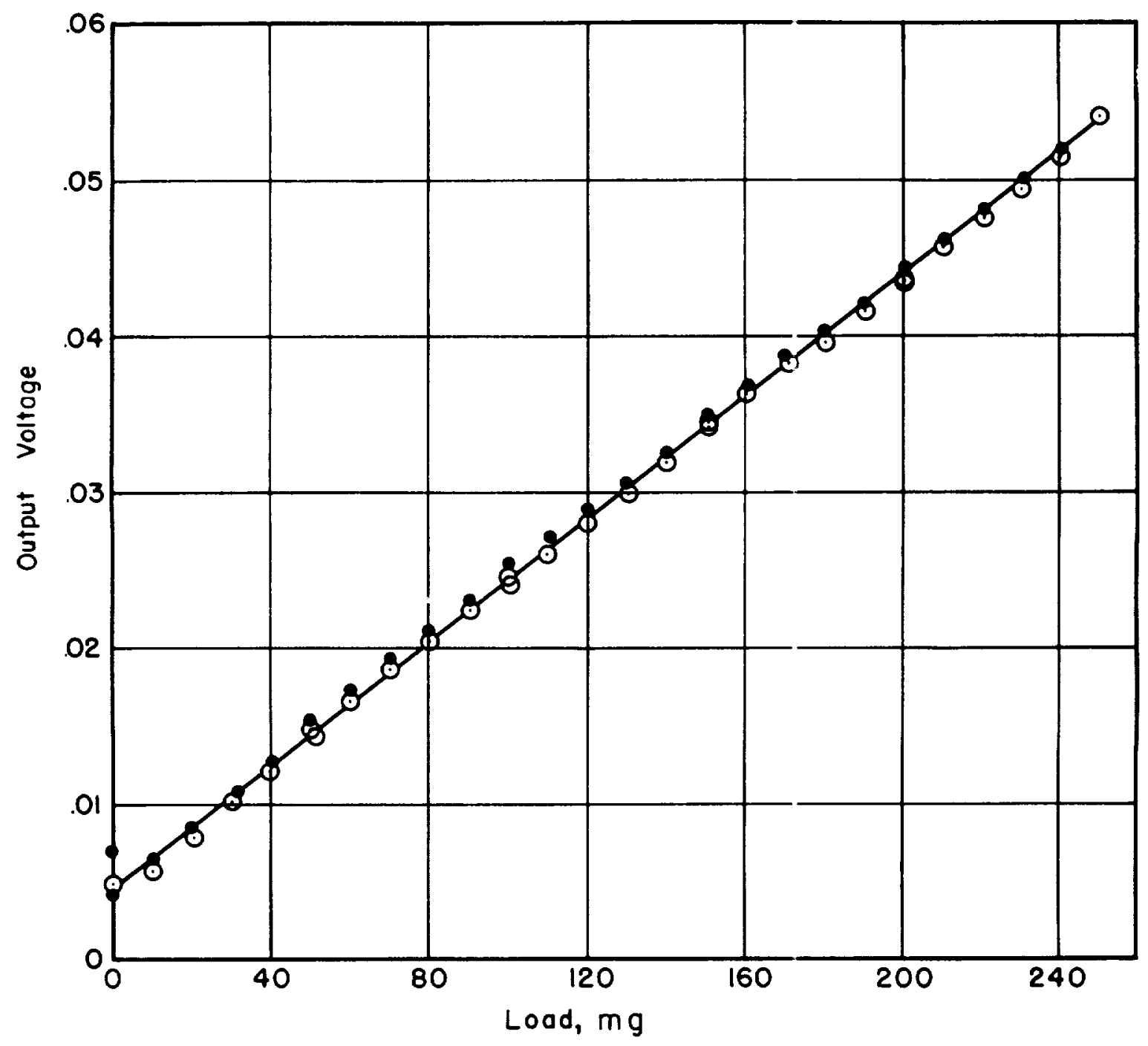

(a) Laminar experiments .

Figure 4.- Calibration of skin-friction meter. 


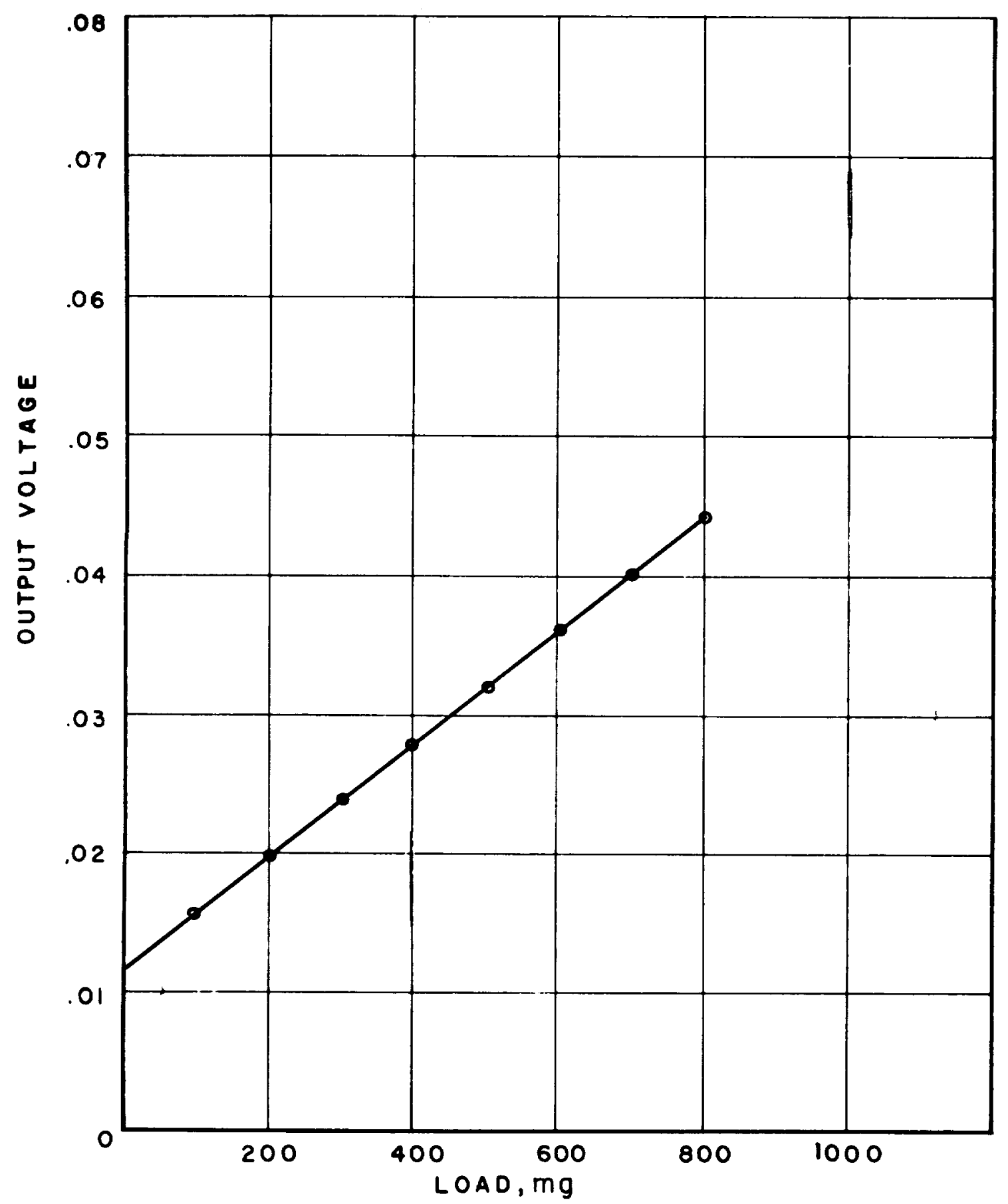

(b) Turbulent experiments.

Figure 4.- Concluded. 


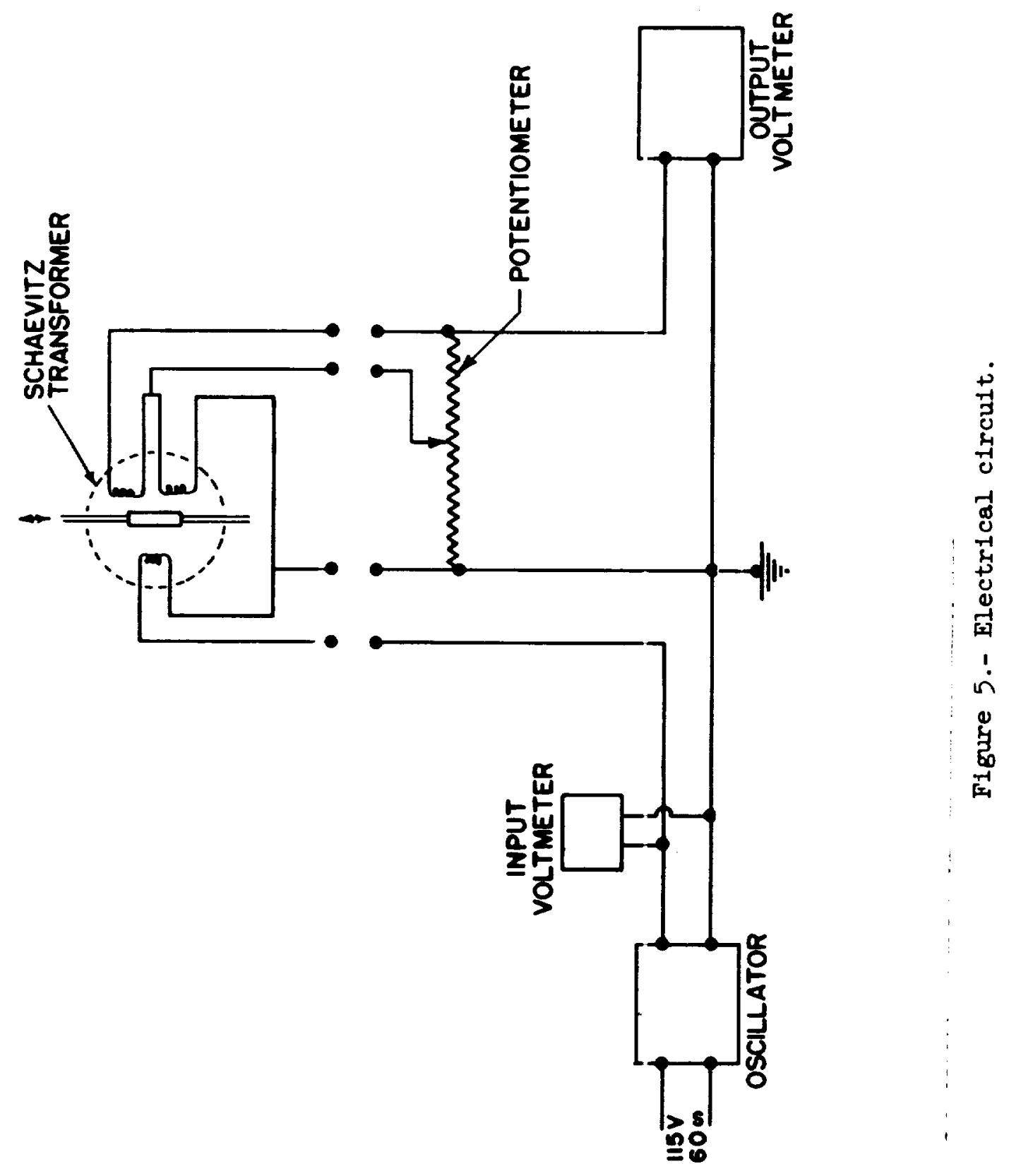




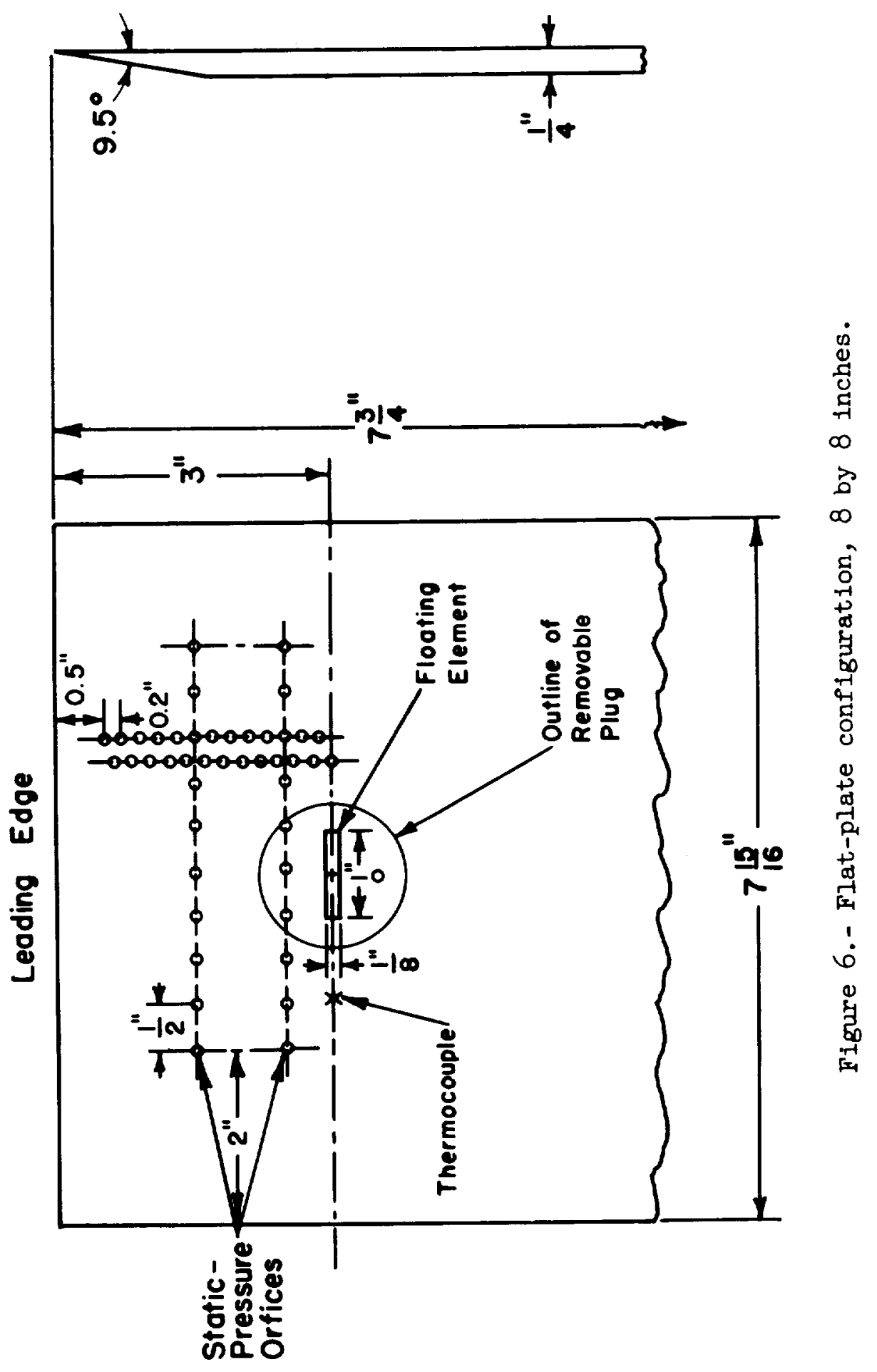




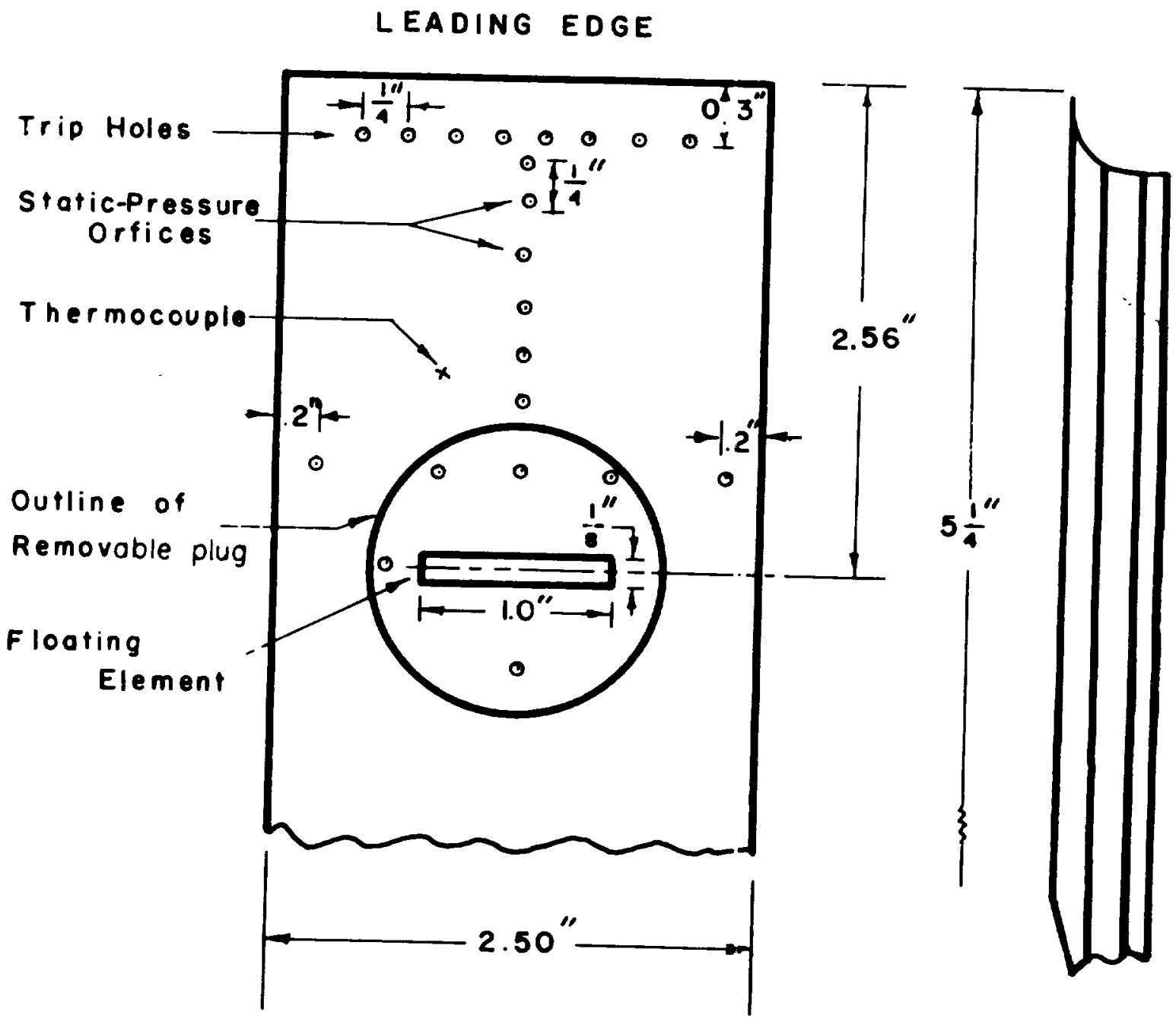

Figure 7.- Flat-plate configuration, 2 by $2 \frac{1}{2}$ inches. 


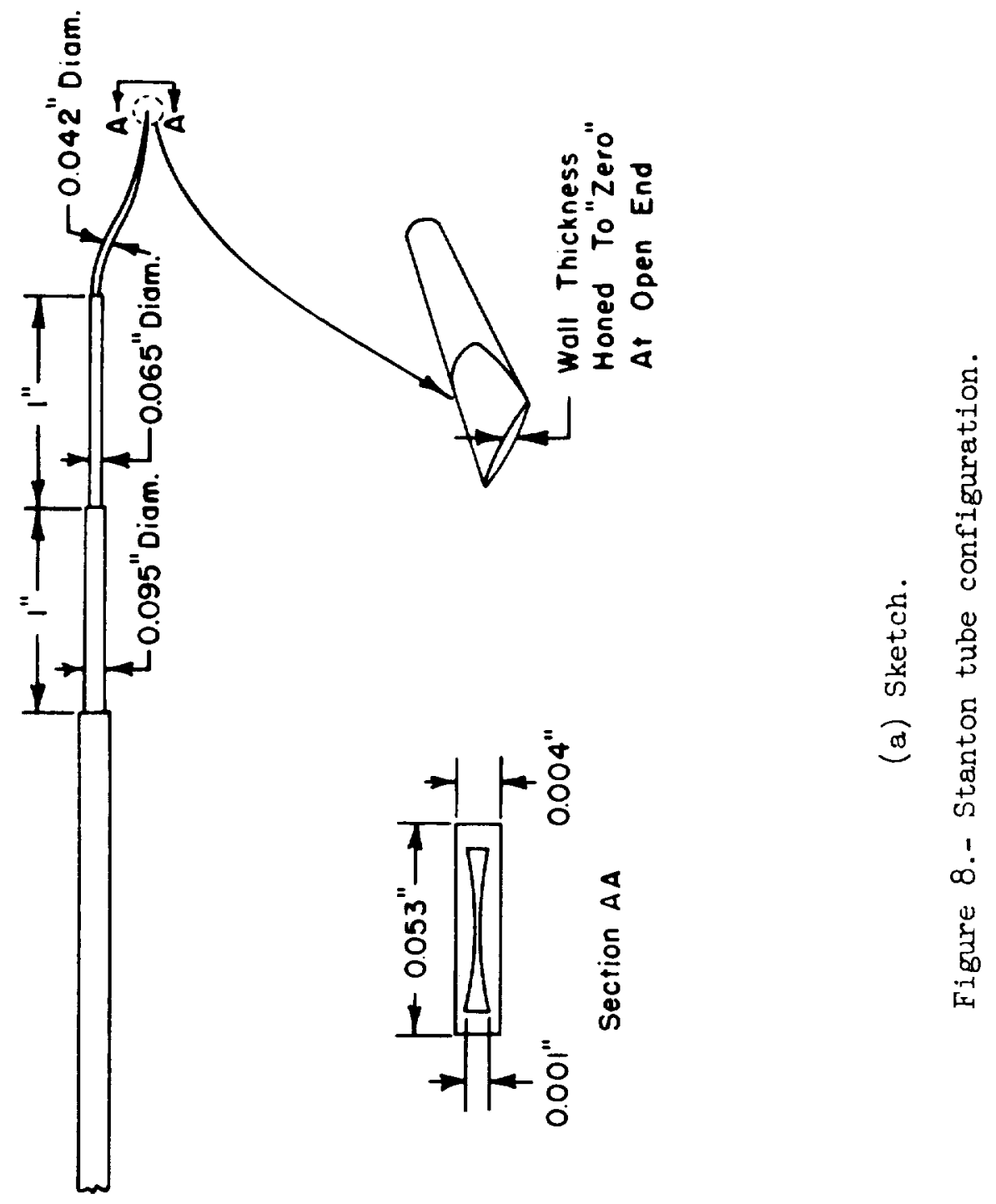




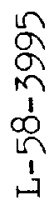

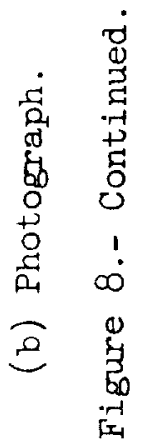




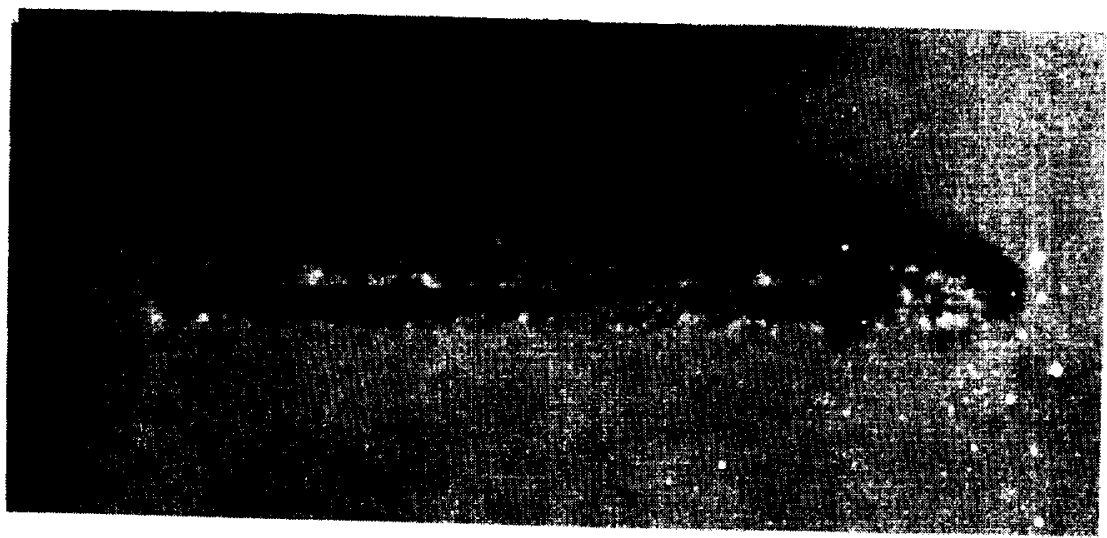

(c) Photomicrograph of head-on L-3996 (c) Photomicrograph of head-on view of probe tip. Figure 8.- Concluded. 


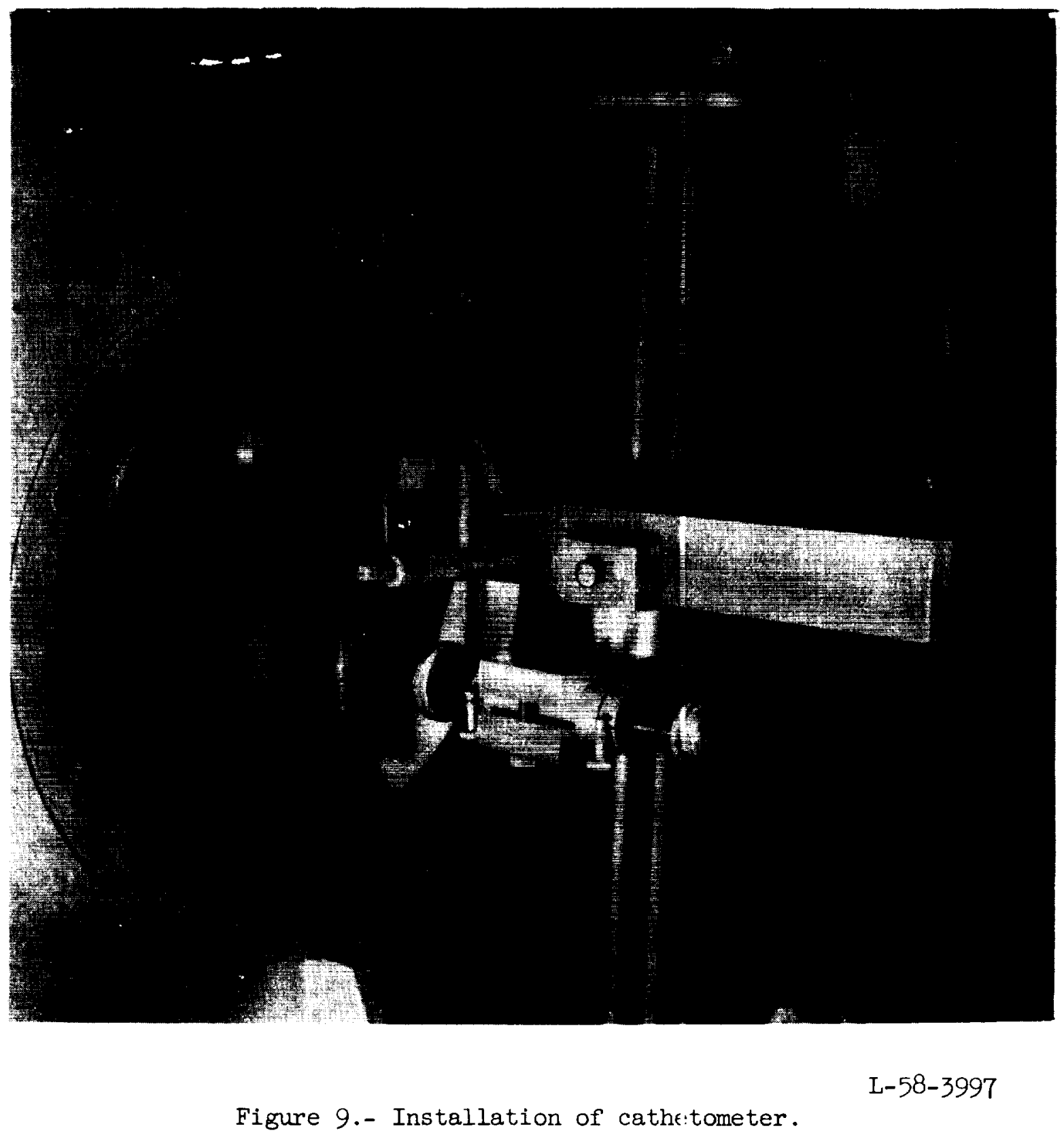




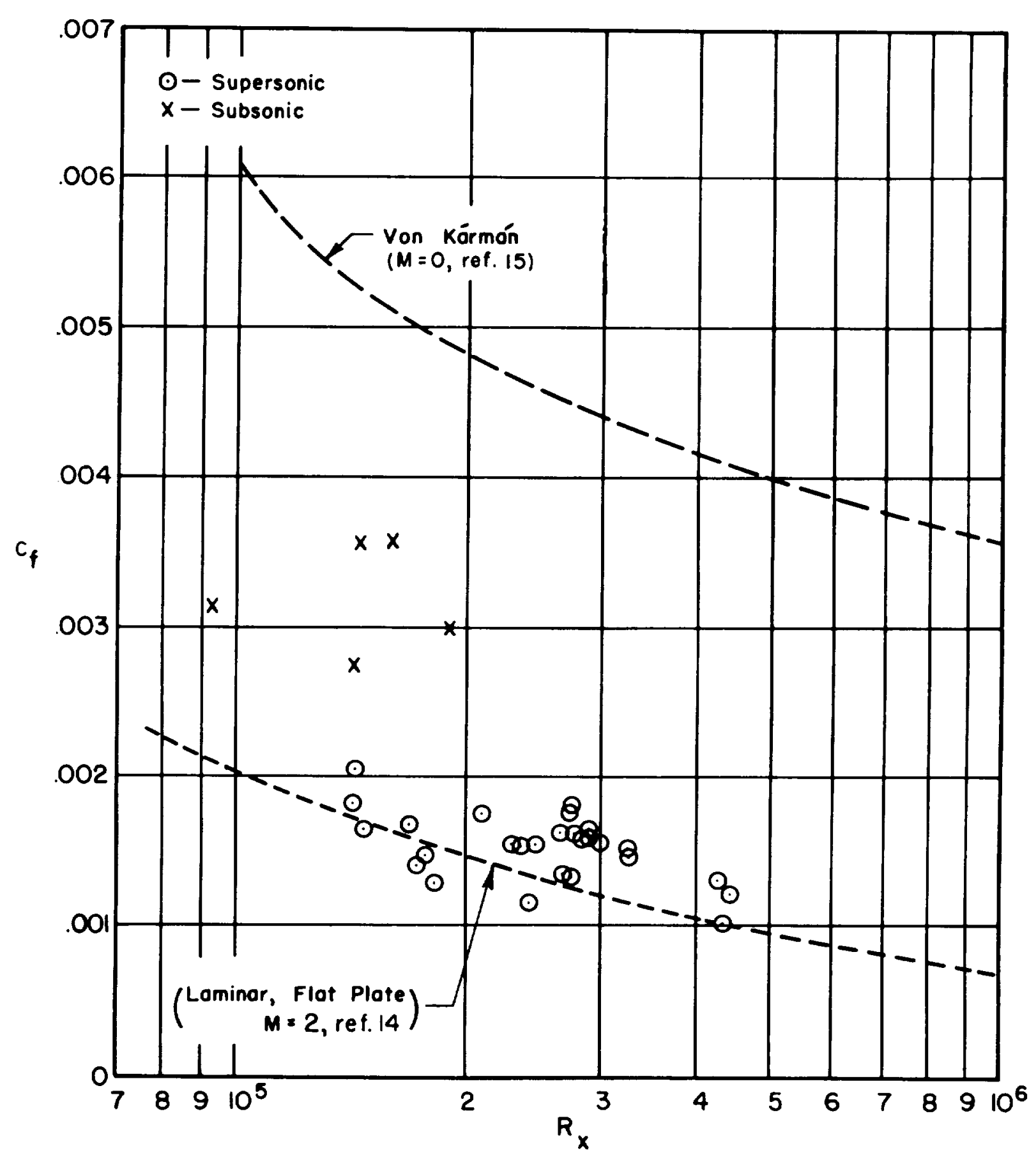

Figure 10.- Local skin friction plotted against Reynolds number measured in 8- by 8-inch tunnel. 
o PRESENT EXPERIMENT, SUPERSONIC FLOW

$\nabla$ HAKKINEN, $M_{\infty}=1.5$ VON KÁRMÁN, INCOMPRESSIBLE FLOW

----- COLES, INCOMPRESSIBLE FLOW

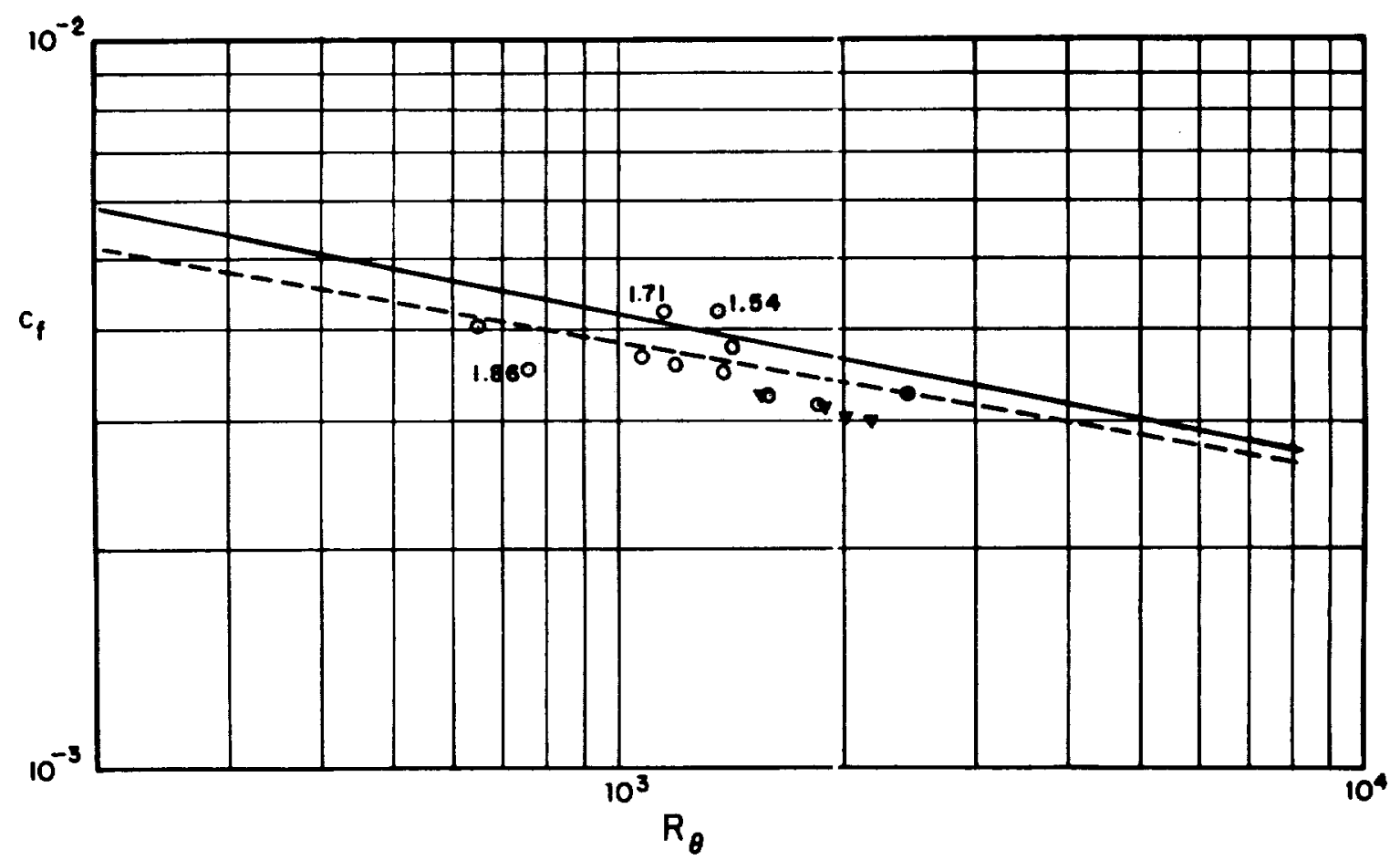

Figure 11.- Local skin friction plotted against momentum-thickness Reynolds number measured in $2-k y 2 \frac{1}{2}-$ inch tunnel. 


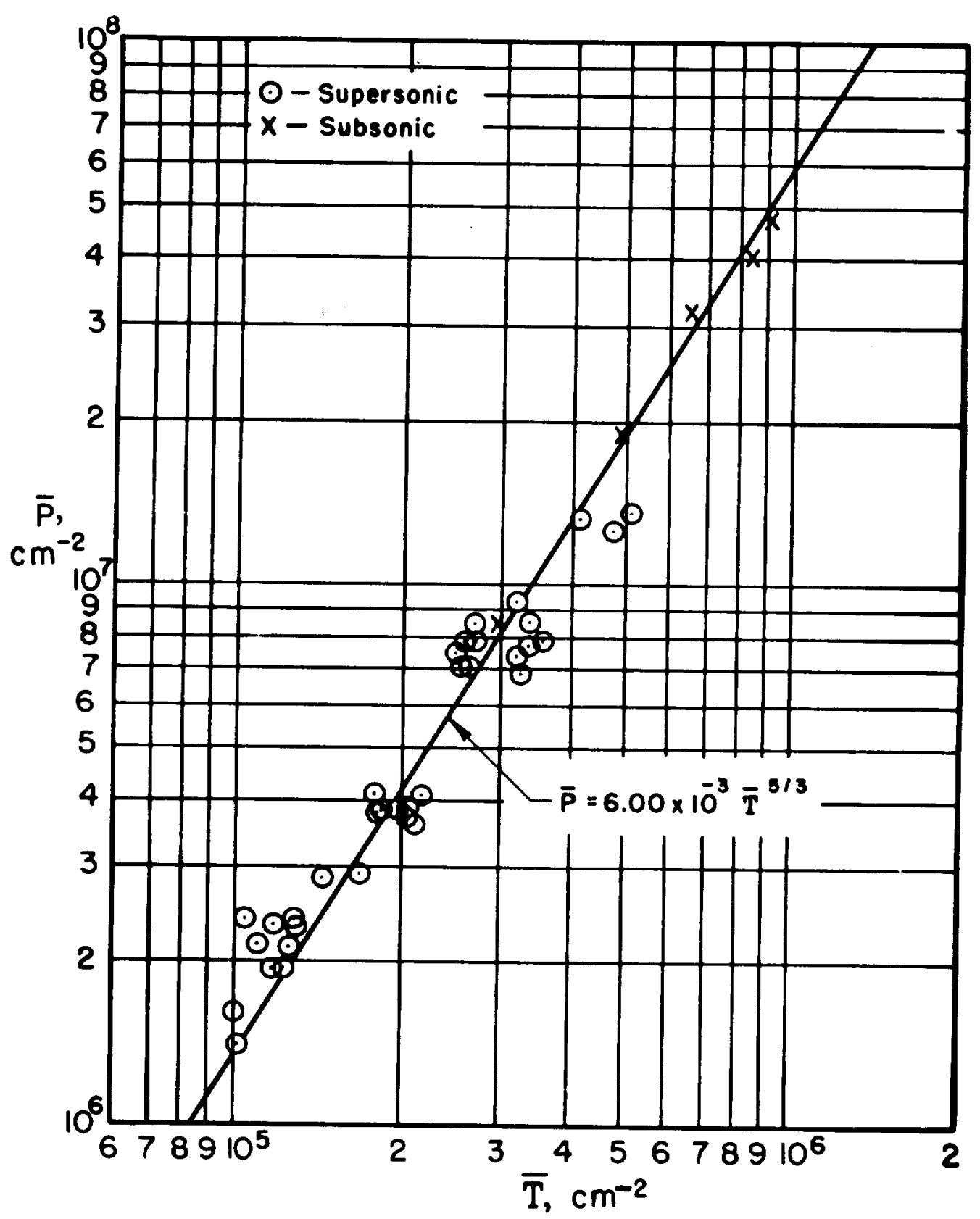

Figure 12.- Stanton tube callbration. $\bar{P} \equiv \frac{\left(P_{T}-P_{O}\right) \rho_{W}}{\mu_{W}{ }^{2}} ; \bar{T} \equiv \frac{T_{W} \rho_{W}}{\mu_{W}}$. 


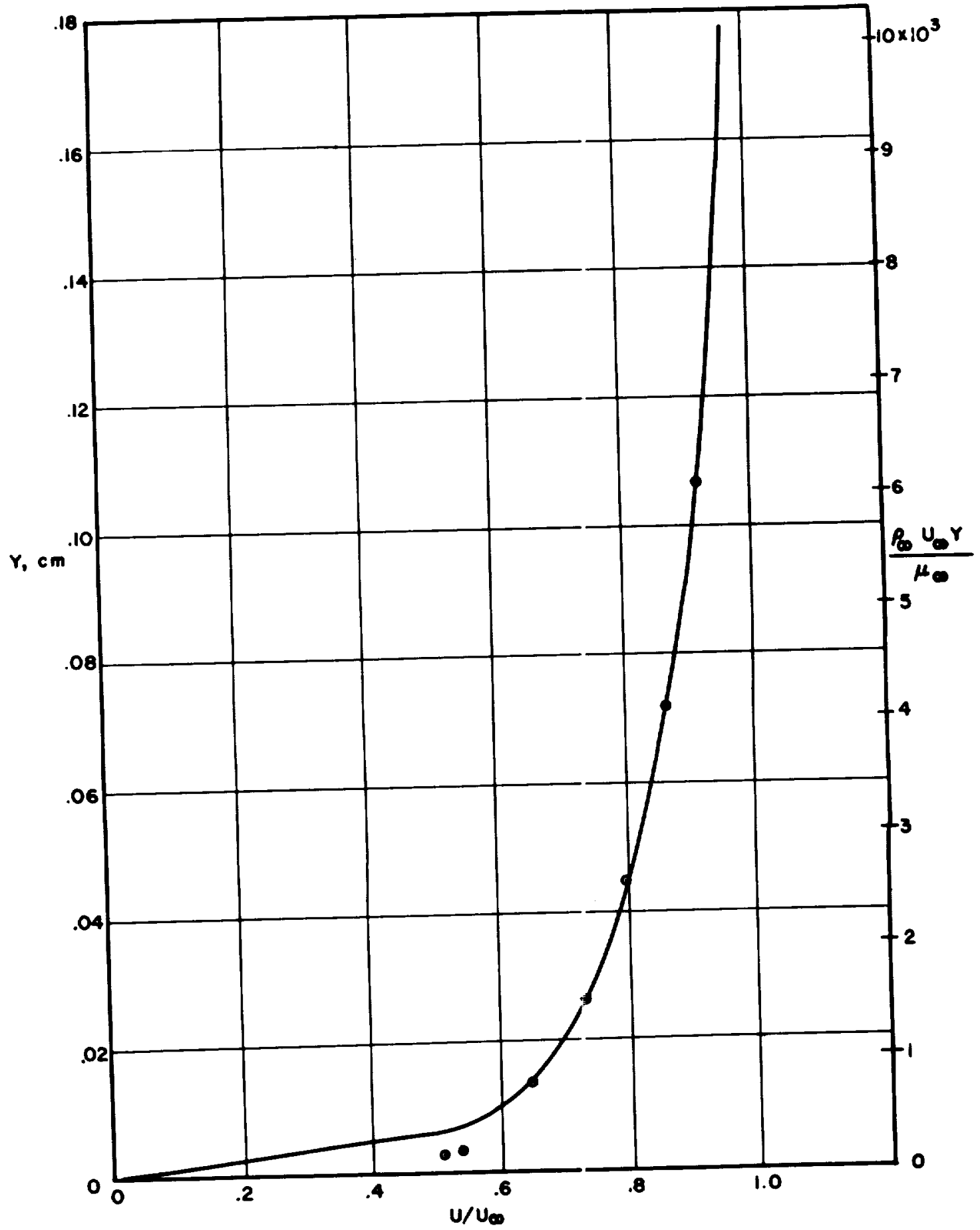

Figure 13.- A typical turbulent velocity profile. $M_{\infty}=1.55$; $R_{x}=372,000$. 


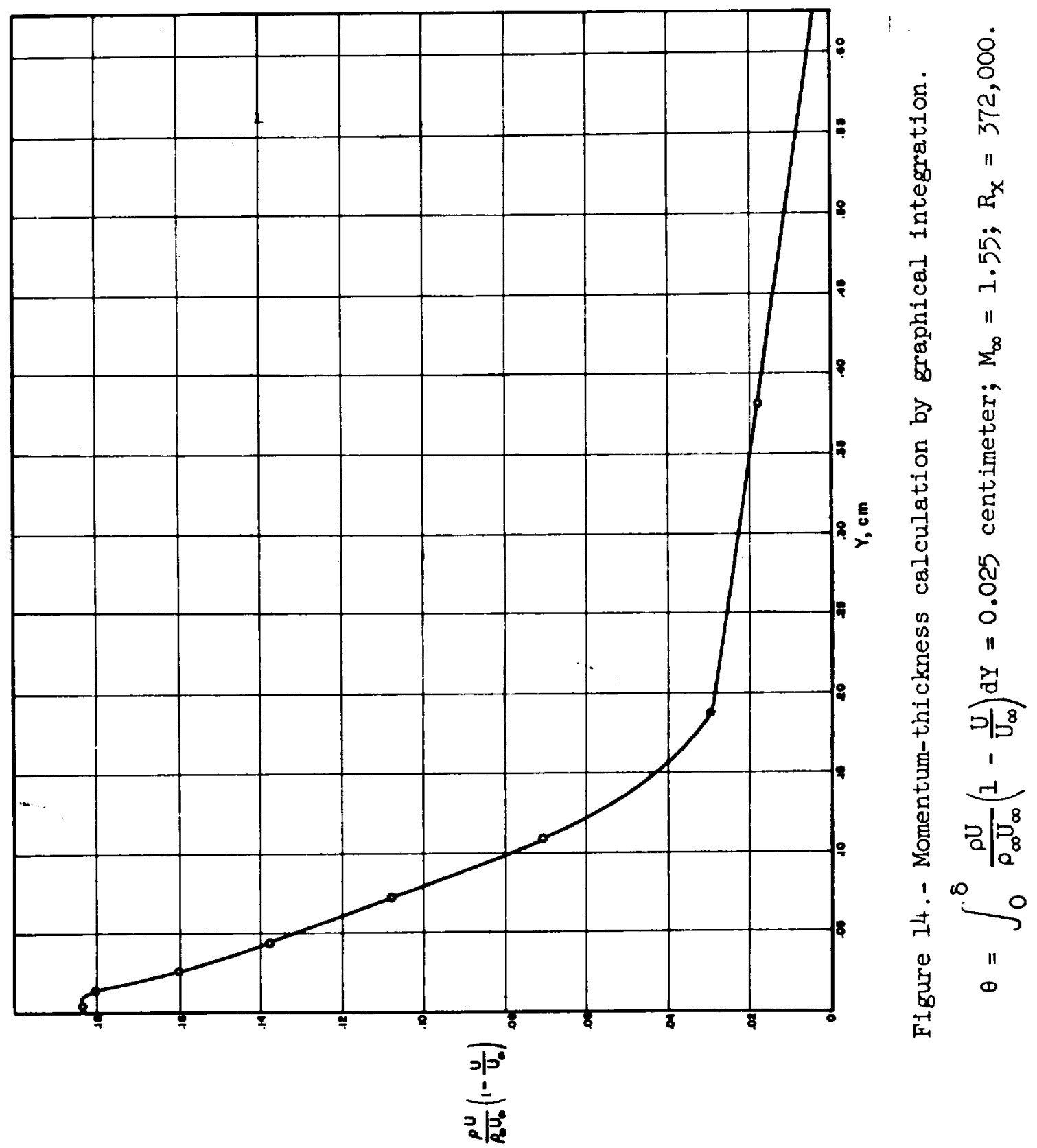




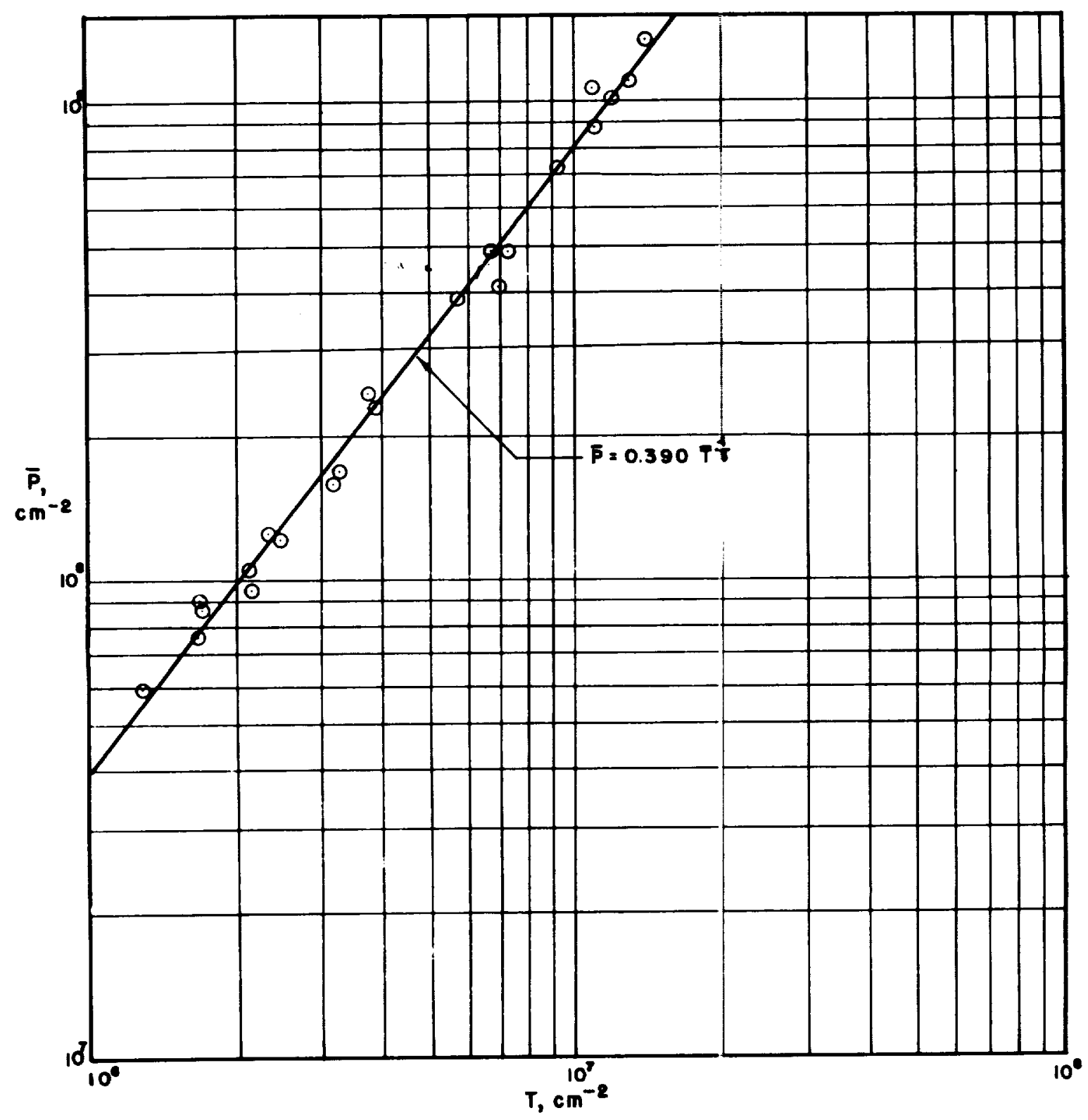

Figure 15.- Stanton tube calibration in turbulent supersonic flow. 


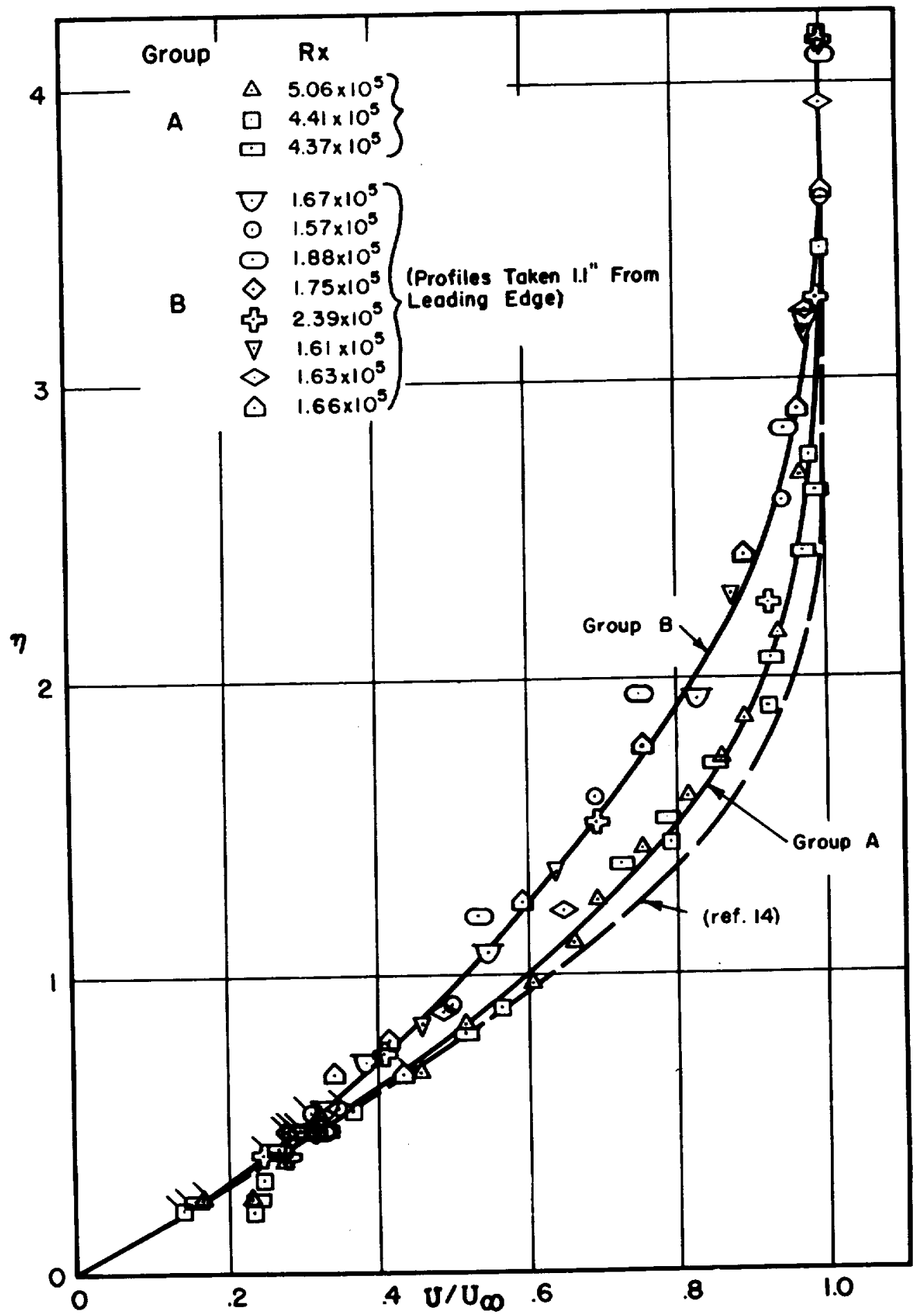

Figure 16.- Laminar velocity profiles. Flagged symbols indicate corrected values at wall. 


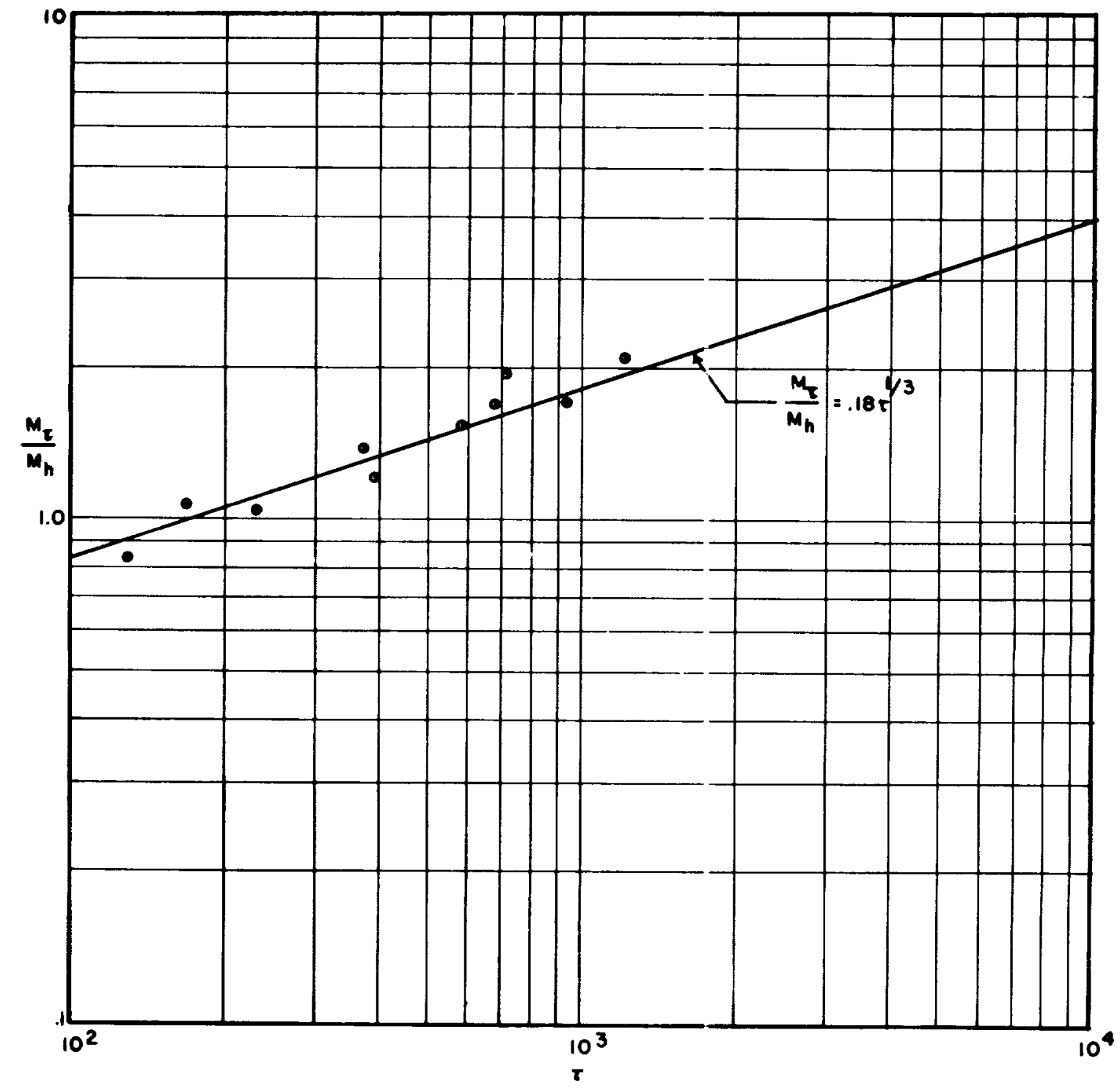

Figure 17.- Deviation from linear profile in turbulent flow as a function of shear parameter $\boldsymbol{T}$. 